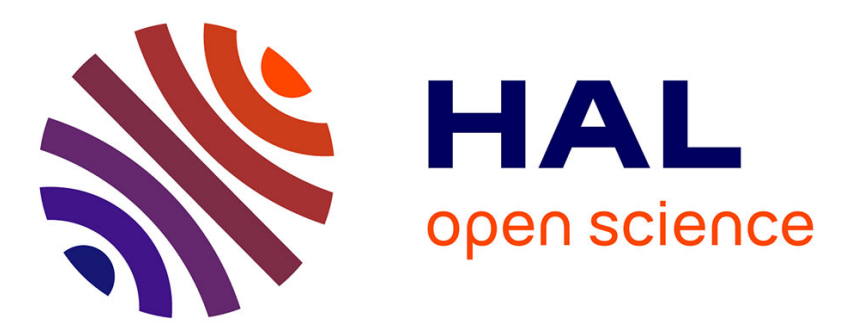

\title{
A switched and scheduled design for model recovery anti-windup of linear plants
} Andrea Cristofaro, Sergio Galeani, Simona Onori, Luca Zaccarian

\section{To cite this version:}

Andrea Cristofaro, Sergio Galeani, Simona Onori, Luca Zaccarian. A switched and scheduled design for model recovery anti-windup of linear plants. European Journal of Control, 2019, 46, pp.23-35. 10.1016/j.ejcon.2018.04.002 . hal-01851129

\section{HAL Id: hal-01851129 \\ https://hal.laas.fr/hal-01851129}

Submitted on 29 Jul 2018

HAL is a multi-disciplinary open access archive for the deposit and dissemination of scientific research documents, whether they are published or not. The documents may come from teaching and research institutions in France or abroad, or from public or private research centers.
L'archive ouverte pluridisciplinaire HAL, est destinée au dépôt et à la diffusion de documents scientifiques de niveau recherche, publiés ou non, émanant des établissements d'enseignement et de recherche français ou étrangers, des laboratoires publics ou privés. 


\title{
A switched and scheduled design for model recovery anti-windup of linear plants
}

\author{
Andrea Cristofaro $^{\mathrm{a}, \mathrm{b}, *}$, Sergio Galeani ${ }^{\mathrm{c}}$, Simona Onori $^{\mathrm{d}}$, Luca Zaccarian ${ }^{\mathrm{f}, \mathrm{e}}$ \\ ${ }^{a}$ School of Science and Technology - Mathematics Division, University of Camerino, 62302 Italy \\ ${ }^{b}$ Department of Engineering Cybernetics, NTNU, Trondheim, 7491 Norway \\ ${ }^{c}$ Dipartimento di Ingegneria Civile e Ingegneria Informatica, University of Rome Tor Vergata, 00133 Italy \\ ${ }^{d}$ Energy Resources Engineering Department, Stanford University, CA 94305, USA \\ ${ }^{e}$ Dipartimento di Ingegneria Industriale, University of Trento, 38123 Italy \\ ${ }_{\text {LAAS-CNRS, Université de Toulouse, CNRS, Toulouse, } 31400 \text { France }}$
}

\begin{abstract}
We provide two nonlinear solutions to the model recovery anti-windup (MRAW) design problem, both of them relying on the definition of a set of nested ellipsoids in the state space of the anti-windup dynamics. Each ellipsoidal set arises from a convenient trade-off between size of the ellipsoid and guaranteed exponential convergence rate induced by the corresponding saturated feedback. The first solution is given by a hybrid selection of the MRAW stabilizer, relying on a natural hysteresis switching mechanism. The second solution corresponds to a Lipschitz but non-differentiable scheduled selection, which essentially smoothens out the discontinuous nature of the nested ellipsoids. We discuss the role of our design architecture and establish a number of important properties induced by the proposed controllers. Their effectiveness is comparatively illustrated on a few example studies.
\end{abstract}

Keywords: model recovery anti-windup, scheduled stabilizer, switched stabilizer, bounded control

\section{Introduction}

Actuator saturation is one of the most frequent hard nonlinearities encountered in control system implementation. When applying any type of control design to a real plant, the control engineer needs to account for the effects of the maximum and minimum control effort allowable for the actuator available on the experimental system. It was observed already from the 1940s that the presence of saturation often caused undesirable behavior and that suitable fixes were necessary to address the arising problem (this fact was actually one of the main motivations for the absolute stability results of the 1950s and the sector properties of static nonlinearities).

A popular approach to deal with saturation, which dates back to the 1960s (see, e.g., [9]), is the so-called anti-windup approach, wherein an "unconstrained controller" is assumed to perform desirably in the absence of saturation (equivalently, when signals are small enough on the saturated closed-loop) and "anti-windup" corrections are necessary for larger signals that would interest the flat region of the saturation, thereby causing performance loss and often instability. The ultimate goals of anti-windup designs are that: 1) the unconstrained behavior imposed by the "unconstrained controller" on the plant is fully reproduced whenever saturation is not activated (namely

\footnotetext{
${ }^{*}$ Corresponding author.

This work is supported by the Research Council of Norway through the Centres of Excellence funding scheme, project No. 223254 - AMOS.

Email addresses: andrea.cristofaro@unicam.it (Andrea

Cristofaro), sergio.galeani@uniroma2.it (Sergio Galeani),

sonori@clemson. edu (Simona Onori), zaccarian@laas.fr (Luca

Zaccarian)
}

for small enough signals); 2) for larger signals the performance is close (as close as possible) to the unconstrained one, and stability is retained as much as possible given the hard limits imposed by the presence of saturation. Initially, anti-windup control was mainly an application driven discipline and most of the results available in the literature were not applicable to general classes of control systems, rather being specific naive solutions for some experimental problems. Toward the end of the 1980s, this lack of formality in the field has been pointed out (see, [8]) and the need of general solutions to the anti-windup augmentation problem led to in an increase of the research effort in the following years. Comprehensive surveys of the first anti-windup solutions are given in $[21,2,28]$. Starting from the mid 1990s, several high performance solutions to the antiwindup problem started to appear. Many of these rely on the socalled reference governor or command governor scheme which is most directly applicable to discrete-time control systems (see, e.g., $[16,1]$ and references therein). Other ones are mostly related to continuous-time cases and rely on the use of linear matrix inequalities (LMIs) together with absolute stability and generalized absolute stability concepts (see, e.g., [31, 18, 25] and references therein). Some very detailed presentations of the above historical overview of anti-windup can be found in the surveys $[14,36]$ and in the two books $[57,35]$.

Among the many aspects that make the anti-windup design challenging is that it inherits well-known intrinsic limitations already known for bounded stabilization. In particular, it was already proved in [34] that global bounded asymptotic stabilization of a linear plant can only be obtained if the plant has eigenvalues in the closed left half plane. Global exponential stabilization via bounded input can then only be obtained if 
the plant is already globally exponentially stable to begin with. Due to this reason, much of the research effort has gone in the direction of giving local guarantees with an intrinsic trade-off between size of the operating region and the aggressiveness of the control law (a notable alternative approach being the one where global nonlinear stabilizers were designed [30, 38]). In this regard, interpolation of controllers has been recognized as a fruitful approach for achieving better regional performances, using e.g. parametrized Riccati equations or switching laws $[50,22,6]$. From the early years of the $21^{\text {st }}$ century, the idea of scheduling different stabilizers was pursued in a number of works [27, 26, 32, 33, 48], and this type of sophisticated generalization of the standard anti-windup paradigm (the one where a simple gain modifies the controller dynamics, driven by the excess of saturation) also led to some different type of anticipatory/delayed anti-windup actions (which can be seen as some kind of multi-stage nonlinear compensation) reported in $[52,32,51,47,46]$ and references therein.

A relevant general approach to the solution of the antiwindup problem is that proposed in the pioneering paper [42] (and the twin paper [43]). In that paper, the authors proposed a framework for anti-windup design wherein the solution to the anti-windup problem (comprising the two properties qualitatively stated above) was obtained from the solution of a simpler external stabilization problem from an input-matched disturbance consisting in the energy that the unconstrained response would have spent outside of the saturation if the saturation limits were not there. That framework was therein denoted as " $\mathcal{L}_{2}$ anti-windup solution" and was shown to solve a specific characterization of the qualitative anti-windup goals listed above, called " $\mathcal{L}_{2}$ anti-windup problem". The parallel thread of work reported in [31, 18, 25] and commented above, aimed indeed at minimizing the input-output $\mathcal{L}_{2}$ gain of the closed loop with anti-windup augmentation, therefore more recently (and also in the book [57]) it was decided to rename this approach "model recovery anti-windup" (MRAW) because through a model-based compensation MRAW is effective at recovering the unconstrained response.

In [42], a preliminary solution was given to the MRAW problem, while indicating that many improvements upon that solution would have been possible via the many degrees of freedom still available in the proposed framework. Then, several extensions have been proposed in later years each of them having different performance and stability guarantees and being applicable to different classes of systems. Among these, [40, 41, 44, 45, 58, 7] report some application studies illustrating the effectiveness of MRAW on practical engineering problems, while in $[39,15]$, the approach was extended to give a nonlinear solution when dealing with exponentially unstable plants, that inevitably lead to non-global results due to the above mentioned intrinsic limitations. MRAW was also extended to rate-saturated plants and applied to relevant flight control problems in [40, 3, 10]. In [54], the same framework was applied to bumpless transfer and this idea was further extended and better characterized in [56]. In [20] the ideas in [42] were extended to the discrete-time case while in [53] they were extended to dead-time plants. A sampled-data implementation of the general approach in [42] was proposed in [4], based on a suitable MPC solution of a discrete-time problem associated to the continuous-time plant. Moreover, a nonlinear scheduled implementation based on hysteresis switching was proposed in [55], although no guarantees were given there on the size of the switching regions.

In this paper, we show that it is possible to adopt multi-stage designs within the MRAW framework, thereby obtaining high performance unconstrained response recovery through the arising aggressive nonlinear anti-windup action. Mimicking the approach in [27, 26, 32, 33], we base the switching/scheduling mechanism on the definition of a suitable set of nested ellipsoids, each of them associated to a choice of stabilizing gains, and then we propose:

1) A hybrid reformulation of the hysteresis switching solution first proposed in [55] and then revised and improved in our preliminary conference paper [12]; as compared to those preliminary results the hybrid formulation provides robust versions of the established asymptotic stability, that stems from the structural properties of the formulation, in addition to a clearer description of the hysteresis mechanism.

2) A scheduling mechanism corresponding to an improved version of the approach proposed in our preliminary work [13, 11]. In particular, as compared to those results, here we choose the feedback gains based on a desirable trade-off between speed of convergence and size of the region of attraction, in addition to well characterizing the nonlinear algebraic loop, already introduced in [54], but assuming a slightly different expression in this new formulation.

Different from [13, 11] where we mainly focus on antiwindup goals, we clarify here that the proposed construction is best characterized as a state-feedback bounded stabilizer, whose ideal application context is the anti-windup one, because in nonlinear model recovery anti-windup, the state of the dynamic augmentation (which must be driven to zero) is available for feedback. We regard this solution as the most advanced MRAW design strategy in terms of trade-off between size of the region of attraction and performance of the control design (which is characterized here in terms of the exponential rate of convergence of the response). As compared to our preliminary results in $[12,13,11]$, we also report here missing proofs, we revise and improve the generation of the ellipsoids that depends here on more intuitive parameters for which we provide a tuning rationale, and the design of the switching/scheduling law. Finally, we illustrate the results on novel examples.

The paper is organized as follows. In Section 2 we propose the construction of the nested ellipsoids establishing the switching/scheduling mechanism proposed subsequently. In Section 3 we show how those ellipsoids can be used for the design of a hysteresis switching stabilizer. Parallel results are given in Section 4 that presents a Lipschitz scheduled stabilizer. All these ingredients are combined in Section 5 that explains how these designs should be incorporated in the MRAW architecture. Finally, Section 6 discusses a few simulation examples with the switching and scheduling approaches. Conclusions and future works are reported in Section 7, while a few technical proofs are reported in the appendix. 
Notation: Given a square matrix $M, \mathrm{He} M:=M+M^{T}$

\section{A family of stabilizers in nested stability regions}

\subsection{Global or regional bounded state feedback stabilization of a linear plant}

Consider the following linear plant:

$$
\dot{x}=A x+B \operatorname{sat}_{\bar{u}}(u),
$$

with state $x \in \mathbb{R}^{n}$ and input $u \in \mathbb{R}^{n_{u}}$ and subject to the symmetric decentralized magnitude saturation nonlinearity $\operatorname{sat}_{\bar{u}}$ with saturation limits $\bar{u}=\left[\begin{array}{lll}\bar{u}_{1} & \cdots & \bar{u}_{n_{u}}\end{array}\right]^{T}$, defined as $\operatorname{sat}_{\bar{u}}(u)=\left[\begin{array}{lll}\operatorname{sat}_{\bar{u}_{1}}\left(u_{1}\right) & \cdots & \operatorname{sat}_{\bar{u}_{n_{u}}}\left(u_{n_{u}}\right)\end{array}\right]^{T}$, where $\operatorname{sat}_{\bar{u}_{i}}(s)=$ $\max \left\{-\bar{u}_{i}, \min \left\{\bar{u}_{i}, s\right\}\right\}, i=1, \ldots, n_{u}$, is the scalar symmetric saturation function with limit $\bar{u}_{i}$.

In this paper we are interested in designing nonlinear switched and scheduled state-feedback control laws for plant (1) capable of inducing high-performance of the arising closed loop. It is well known (see, e.g., [34]) that plant (1) is globally stabilizable from the bounded input if and only if $A$ has no eigenvalue with positive real part (namely, the open-loop dynamics is not exponentially unstable). Due to this reason, we start off our design by focusing on a suitable stabilizer taking the form

$$
u=K x+L \operatorname{sat}_{m}(u)
$$

and ensuring a reasonably large domain of attraction, depending on the open-loop dynamics of (1). Stabilizer (2) requires the solution of a nonlinear algebraic loop, which is rather simple with a small number of inputs is at hand but may become rather complicated in the presence of many saturated inputs (see [57, §2.3.7] for a discussion about how to solve these algebraic loops). The algebraic loop has been shown to generally lead to improved responses (see, e.g., [31]) but can be easily avoided (at the expense of a potentially deteriorated performance) by fixing $X_{2}=0$ in the optimization presented below in (3). In this special case, variants of the corresponding results have been presented in [19] (see also [35]). The following proposition provides a design guideline for the selection of the gains in (2).

Proposition 1. Consider the dynamical system (1), a scalar $\lambda \geq 0$, and the following set of LMIs in the variables $\left\{Q, X_{1}, X_{2}, U, Y, \rho^{-2}\right\}$ :

$$
\begin{aligned}
& Q=Q^{T} \geq \rho^{2} I, U>0 \text { diagonal } \\
& \operatorname{He}\left[\begin{array}{cc}
(A+\lambda I) Q+B X_{1} & B\left(X_{2}-U\right) \\
X_{1}+Y & X_{2}-U
\end{array}\right]<0, \\
& {\left[\begin{array}{cc}
m_{k}^{2} & Y^{(k)} \\
Y^{(k)^{\prime}} & Q
\end{array}\right] \geq 0, \quad k=1, \ldots, n_{u},}
\end{aligned}
$$

where $Y^{(k)}$ denotes the $k$-th row of matrix $Y$.

Then given any solution to (3), the feedback stabilizer (2) with parameters $(K, L)=\left(K_{1}, L_{1}\right)$ with

$$
\begin{aligned}
K_{1} & =\left(I-X_{2} U^{-1}\right)^{-1} X_{1} Q^{-1}, \\
L_{1} & =-\left(I-X_{2} U^{-1}\right)^{-1} X_{2} U^{-1},
\end{aligned}
$$

guarantees 1) well-posedness of the algebraic loop in (2), namely existence of a unique (nonlinear) globally Lipschitz. function $\varphi$ such that $u=\varphi(x) ; 2$ ) exponential stability of the origin with exponential rate $\lambda_{1}=\lambda$ for (1), (2) with basin of attraction larger than the set (where we use $Q_{1}=Q$ ):

$$
\mathcal{E}_{1}:=\mathcal{E}\left(Q_{1}^{-1}\right):=\left\{x^{T} Q_{1}^{-1} x \leq 1\right\}
$$

which is forward invariant and contains the ball $\rho \mathcal{B}=\{|x| \leq \rho\}$; 3) global exponential stability of the origin with exponential rate $\lambda$, if $Y=0$.

Moreover, the following can be proven about the feasibility of (3):

1. If the open-loop dynamics (1) is exponentially stable with exponential rate larger than $\lambda$ (namely all eigenvalues of $A$ have real part strictly smaller than $-\lambda$ ), then LMIs (3) are feasible with $Y=0$, which guarantees global exponential stability.

2. If the open-loop dynamics (1) has exponential rate not smaller than $\lambda$ (namely all eigenvalues of A have real part smaller than or equal to $-\lambda)^{1}$, then LMIs (3) are feasible for any (arbitrarily large) value of $\rho$.

3. In all cases, LMIs (3) are feasible for a sufficiently small value of $\rho$.

Remark 1. (Different optimization goals) While the focus of Proposition 1 is mostly on the size of the domain of attraction estimate (called stability region henceforth), it should be recognized that increasing the value of the certified exponential rate $\lambda$ will in general reduce the size of the stability region so that a suitable trade-off should be performed. The goal of the switching and scheduled solutions in this paper is to partially overcome this trade-off by suitably increasing the performance level $\lambda$ as the plant state gets closer to the origin.

In conditions (3) and throughout the rest of this paper, we focus on the optimality criterion associated to the exponential rate $\lambda$ in (3b). However, different performance metrics can be also optimized using the approach proposed here. For example, in [12] the goal was the minimization of the $\mathcal{L}_{2}$ gain from an exogenous input $w$ to a performance output $z$ of an extended plant dynamics of the form:

$$
\begin{aligned}
\dot{x} & =A x+B \operatorname{sat}_{\bar{u}}(u)+B_{w} w \\
z & =C x+D \operatorname{sat}_{\bar{u}}(u)+D_{w} w .
\end{aligned}
$$

This different performance goal can then be optimized by minimizing the $\mathcal{L}_{2}$ gain estimate $\gamma$ and replacing condition (3) by the conditions in [12, eqs. (7)]. This modification affects many of the derivations in the rest of this section, but the corresponding results are a straightforward extension of the techniques developed here.

Proof of Proposition 1. Proposition 1 is a slight modification of the results presented in [12, §II.B] and then recalled in [13,

\footnotetext{
${ }^{1}$ Since $\lambda \geq 0$, this implies that $A$ has no eigenvalues with positive real part.
} 
Prop. 2]. The proof follows standard LMI derivations based on the generalized sector conditions proposed in [19, 24] and corresponding to the fact that for any pair of inputs $u, w \in \mathbb{R}^{n_{u}}$ and any diagonal positive matrix $W>0$, the following holds:

$$
\mathrm{dz}(w)=0 \Rightarrow \mathrm{dz}(u)^{T} W(u-\mathrm{dz}(u)+w) \geq 0,
$$

where $\mathrm{dz}(u):=u-\operatorname{sat}(u)$. In order to exploit condition (6), we may rewrite the plant input selection in (2) as follows:

$$
\begin{aligned}
u & =(I-L)^{-1} K x-(I-L)^{-1} L \mathrm{dz}(u) \\
& =X_{1} Q^{-1} x+X_{2} U^{-1} \mathrm{dz}(u)
\end{aligned}
$$

where the second equality can be verified by inverting (4) and doing some straightforward simplifications. The following relation is then straightforward from (7):

$$
\begin{aligned}
\dot{x} & =A x+B \operatorname{sat}_{\bar{u}}(u) \\
& =\left(A+B X_{1} Q^{-1}\right) x+B\left(X_{2} U^{-1}-I\right) \mathrm{dz}(u) .
\end{aligned}
$$

Consider now formulation (7), (8) of the closed loop and notice that using the Lyapunov function candidate $V(x)=x^{T} Q^{-1} x$ and selecting $w=H x$, with $H$ to be specified, and $W=U^{-1}$ in (6), we may write:

$$
\mathrm{dz}(H x)=0 \Rightarrow \dot{V}(x) \leq \dot{V}(x)+2 \mathrm{dz}(u)^{T} U^{-1}(u-\mathrm{dz}(u)+H x),
$$

whose rightmost term can be written as:

$$
2\left[\begin{array}{c}
x \\
\mathrm{dz}(u)
\end{array}\right]^{T}\left[\begin{array}{cc}
Q^{-1}\left(A+B X_{1} Q^{-1}\right) & Q^{-1} B\left(X_{2} U^{-1}-I\right) \\
U^{-1}\left(X_{1} Q^{-1}+H\right) & U^{-1}\left(X_{2} U^{-1}-I\right)
\end{array}\right]\left[\begin{array}{c}
x \\
\mathrm{dz}(u)
\end{array}\right]
$$

where the first block line contains terms arising from $\dot{V}$ and the second block line contains terms from the generalized sector conditions. Pre- and post-multiplying the matrix appearing in (10) by $\operatorname{diag} Q, U$ and selecting $H=Y Q^{-1}$, we obtain that inequality (3b) implies

$$
\mathrm{dz}\left(Y Q^{-1} x\right)=0 \Rightarrow \dot{V}(x) \leq-2 \gamma V(x) .
$$

Consider now inequality (3c), and note that, with the selections above, it implies:

$$
\left|m_{k}^{-1} H^{(k)} x\right|^{2} \leq x^{T} Q^{-1} x, \quad \forall k=1, \ldots, n_{u},
$$

which, combined with (11), implies

$$
V(x) \leq 1 \Rightarrow \mathrm{dz}(H x)=0 \Rightarrow \dot{V}(x) \leq-2 \gamma V(x),
$$

thus clearly proving items (2) and (3) of the statement from standard comparison and Lyapunov theorems. Moreover, let $x$ belong to $\rho \mathcal{B}$, i.e. $|x|^{2} \leq \rho^{2}$. Then, from $Q^{-1} \leq \rho^{-2} I$, the following chain of inequalities holds

$$
x^{T} Q^{-1} x \leq x^{T} \rho^{-2} I x=\rho^{-2}|x|^{2} \leq 1,
$$

thus showing the inclusion $\rho \mathcal{B} \subseteq \mathcal{E}_{1}$.

Regarding item (1), the proof is based on the following technical results
Lemma 1. Given a positive-definite diagonal matrix $W \in$ $\mathbb{R}^{n_{u} \times n_{u}}$ and a square matrix $S \in \mathbb{R}^{n_{u} \times n_{u}}$ with $\operatorname{det}(I-S) \neq 0$, if $2 W+W S(I-S)^{-1}+\left(I-S^{T}\right)^{-1} S^{T} W>0$, then $I-S \Delta$ is nonsingular for all matrices $\Delta$ included in the set

$$
O_{n_{u}}:=\left\{\Delta: \Delta=\operatorname{diag}\left(\delta_{1}, \ldots, \delta_{n_{u}}\right), \delta_{k} \in[0,1] \forall k\right\} .
$$

Lemma 2. Consider a locally Lipschitz map $F: \mathbb{R}^{n} \rightarrow \mathbb{R}^{n}$ and assume that the differential inclusion $J F(z) \in \mathcal{M}$ holds for a.e. $z \in \mathbb{R}^{n}$, where $J F(z)$ denotes the Jacobian of $F$ and $\mathcal{M} \subset \mathbb{R}^{n \times n}$ is a compact and convex subset such that $\operatorname{det}(M) \neq 0 \forall M \in \mathcal{M}$. Then there exists a unique globally Lipschitz function $G: \mathbb{R}^{n} \rightarrow$ $\mathbb{R}^{n}$ with $F(G(z))=z \forall z \in \mathbb{R}^{n}$.

The proof of Lemma 1 is given in Appendix A, while the proof of Lemma 2 can be found in [54, Prop. 2]. Let us focus now on item (1). From the LMI (3b) one can infer that

$$
X_{2}+X_{2}^{T}-2 U<0
$$

as the bottom right term of the matrix must be negative-definite. On the other hand, it follows from (4) that the matrix $X_{2}$ satisfies

$$
X_{2}=-L_{1}\left(I-L_{1}\right)^{-1} U
$$

and hence inequality (13) can be rewritten in terms of $L_{1}$ as

$$
-L_{1}\left(I-L_{1}\right)^{-1} U-U\left(I-L_{1}^{T}\right)^{-1} L_{1}^{T}-2 U<0
$$

or equivalently as

$$
-W L_{1}\left(I-L_{1}\right)^{-1}-\left(I-L_{1}^{T}\right)^{-1} L_{1}^{T} W-2 W<0
$$

where we have set $W=U^{-1}$. Consider the map $F(u):=$ $u-L_{1} \operatorname{sat}_{\bar{u}}(u)$ and observe that, since the derivative of each component of the saturation function is either 0 or 1 a.e., the inclusion

$$
\begin{aligned}
J F(u) & \in \overline{c o}\left(\left\{I-L_{1} \operatorname{diag}\left(\delta_{1}, \ldots, \delta_{n_{u}}\right), \delta_{k} \in\{0,1\} \forall k\right\}\right) \\
& =\left\{I-L_{1} \Delta, \Delta \in O_{n_{u}}\right\}=: \mathcal{M}
\end{aligned}
$$

holds, where $\overline{c o}(\mathcal{E})$ denotes the closed convex hull of the set $\mathcal{E}$ and $\mathcal{O}_{n_{u}}$ is defined in Lemma 1. Due to (14), the conditions of Lemma 1 are met and thus the set $\mathcal{M}$, which is compact and convex by construction, contains nonsingular matrices only. The conclusion follows then by applying Lemma 2, which establishes that there exists a unique globally Lipschitz map $G(z)$ with $F(G(z))=z$ and

$$
\varphi(x)-L_{1} \varphi(x)=K_{1} x,
$$

where we have set $\varphi(x)=G\left(K_{1} x\right)$.

Only the feasibility of the LMIs remains to be addressed. Since by assumption the plant is stabilizable, there exist a feedback gain $K_{0}$ and two positive definite matrices, $Q_{0}=Q_{0}^{T}>0, R_{0}=$ $R_{0}^{T}>0$, with

$$
\operatorname{He}\left[(A+\lambda I) Q_{0}+B K_{0} Q_{0}\right]=-R_{0} .
$$

Selecting $Q=v Q_{0}, Y=K_{0} Q=v K_{0} Q_{0}, X_{1}=-Y+\left(X_{2}-\right.$ $U)^{T} B^{T}$ and $2 U>X_{2}^{T}+X_{2}$, it can be easily seen that inequality 
(3b) is satisfied for any $v>0$ because the left-hand side matrix becomes block diagonal. In the case of open-loop exponential stability (item (1)), the inequalities are trivially satisfied with the choice

$$
\begin{gathered}
Y=0, X_{1}^{T}=-B\left(X_{2}-U\right), \rho^{2}=\sigma_{\min }(Q), \\
2 U>X_{2}+X_{2}^{T}, \operatorname{He}[(A+\lambda I) Q]<0 .
\end{gathered}
$$

Regarding item (3), observe that condition (3c) is equivalent to

$$
m_{k}^{2}-v Y_{0}^{(k)} Q_{0}^{-1} Y_{0}^{(k)^{\prime}} \geq 0 \quad k=1, \ldots, n_{u}
$$

where we have denoted by $Y_{0}^{(k)}$ the $k^{\text {th }}$-row of $Y_{0}$. Then, it is sufficient to pick

$$
v \leq \underline{v}:=\min _{k=1, \ldots, n_{u}} \frac{m_{k}^{2}}{Y_{0}^{(k)} Q_{0}^{-1} Y_{0}^{(k)^{\prime}}}
$$

which indeed implies the feasibility for any $\rho^{2} \leq v \sigma_{\min }\left(Q_{0}\right)$. Item (2) corresponds to semi-global stabilization, and requires a few more manipulations. Define $A_{\lambda}=A+\lambda I$ and note that by assumption it has eigenvalues in the closed left half plane. Following [25, Proposition 2], it can be proven that there exists $\gamma_{0}>0$ such that, for any $\zeta>0$ there exists $Q=Q^{T}>\zeta I$ with

$$
\operatorname{He}\left[\begin{array}{cc}
A_{\lambda} Q-\frac{\gamma_{0}^{2}}{2} B B^{T} & -\frac{B}{2} \\
0 & -\frac{I}{2}
\end{array}\right]<0 .
$$

Therefore, setting $Y=-\left(\gamma_{0}^{2} / 2\right) B^{T}, X_{1}=-Y, U=X_{2}+I / 2$, where $X_{2}$ is an arbitrary diagonal matrix with $X_{2}>-I / 2$, it is easily checked that $(3 b)$ is satisfied because it coincides with (15). Moreover, also inequalities (3a) and (3c) are satisfied by selecting

$$
\zeta \geq \max \left\{\rho^{2}, \max _{k \in\left\{1, \ldots, n_{u}\right\}}\left(\frac{\left|Y^{(k)}\right|}{m_{k}}\right)\right\}
$$

in (15)

\subsection{Generation of nested stabilizers}

Proposition 1 is used in this section as a starting point for the generation of a family of $N$ nested ellipsoids $\mathcal{E}_{1} \supset \cdots \supset \mathcal{E}_{N}$, each one of them associated with a pair of gains $\left(K_{i}, L_{i}\right)$ to be used in control law (2), and ensuring exponential stability of the origin from the corresponding ellipsoid. In particular, the region $\mathcal{E}_{1}$ in (5) corresponds to the largest of these ellipsoids and an algorithm is proposed to generate a number of nested ellipsoids within $\mathcal{E}_{1}$. The reason for designing several gains in nested sets is that the closer the trajectory is to the origin, the higher gain can be used with stability guarantees, thereby improving the small and medium signal performance of the stabilizer. The solid and bold level sets in Figure 1 represent an example of two nested sets arising from the algorithm proposed in this section. This construction has common features with that of [55], even though no generalized sector conditions where used in that work, thereby resulting in a conservative design.
Moreover, the construction is a suitable combination of our preliminary results in [12] (where a switching law was used) and $[11,13]$ (where scheduled laws were used and a similar algorithm to the one reported here is proposed). Here, once the family of nested sets with the corresponding gains is in place, we present switched and scheduled nonlinear stabilizers in Sections 3 and 4, respectively.

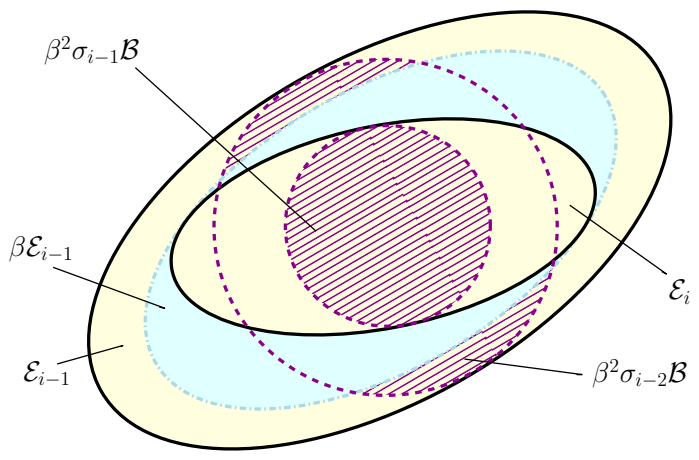

Figure 1: Two nested level sets arising from Algorithm 1.

Given the outer ellipsoid $\mathcal{E}_{1}$ as per (5), the proposed nested regions construction is given in Algorithm 1, which is based on two key parameters:

1. a shrinking factor $\beta \in(0,1)$ providing outer and inner constraints for set $\mathcal{E}_{i}$ graphically represented in Figure 1, based on set $\mathcal{E}_{i-1}$ and corresponding to (16c) for the outer constraint (dashed-dotted boundary in Figure 1) and to (16d) for the inner constraint (dashed boundary in Figure 1). Figure 1 reports in bold the shape of set $\mathcal{E}_{i}$ that respects these constraints, given the set $\mathcal{E}_{i-1}$ reported in thin solid line;

2. a linearity radius $\eta>0$ specifying a ball $\eta \mathcal{B}=\{|x| \leq \eta\}$, where the nested control design should provide a linear feedback. For the algorithm to make sense it is necessary that $\eta \mathcal{B}$ is contained in the interior of $\mathcal{E}_{1}$, which holds if and only if $\eta^{2}<\sigma_{\min }\left(Q_{1}\right)$.

The following result establishes desirable features of Algorithm 1 .

Proposition 2. Consider any solution $\left(\lambda_{1}, Q_{1}, K_{1}, L_{1}\right)$ arising from the construction in Proposition 1 (see (4) and (5)), any shrinking factor $\beta \in(0,1)$ and any $\eta>0$ satisfying $\eta^{2} \leq$ $\sigma_{\min }\left(Q_{1}\right)$.

Then Algorithm 1 always terminates in a finite number $N$ of steps and guarantees:

$$
\begin{gathered}
\mathcal{E}_{1} \supset \mathcal{E}_{2} \cdots \supset \mathcal{E}_{N}, \\
\lambda_{1} \leq \lambda_{2} \cdots \leq \lambda_{N} .
\end{gathered}
$$

Moreover, for each $i \in\{1, \ldots, N\}$, the closed loop (1), (2) with $K=K_{i}$ and $L=L_{i}$ has a well posed algebraic loop, is exponentially stable to the origin, with basin of attraction containing the set

$$
\mathcal{E}_{i}=\left\{x^{T} Q_{i}^{-1} x \leq 1\right\},
$$

and is also such that the set $\mathcal{E}_{i}$ is forward invariant. 


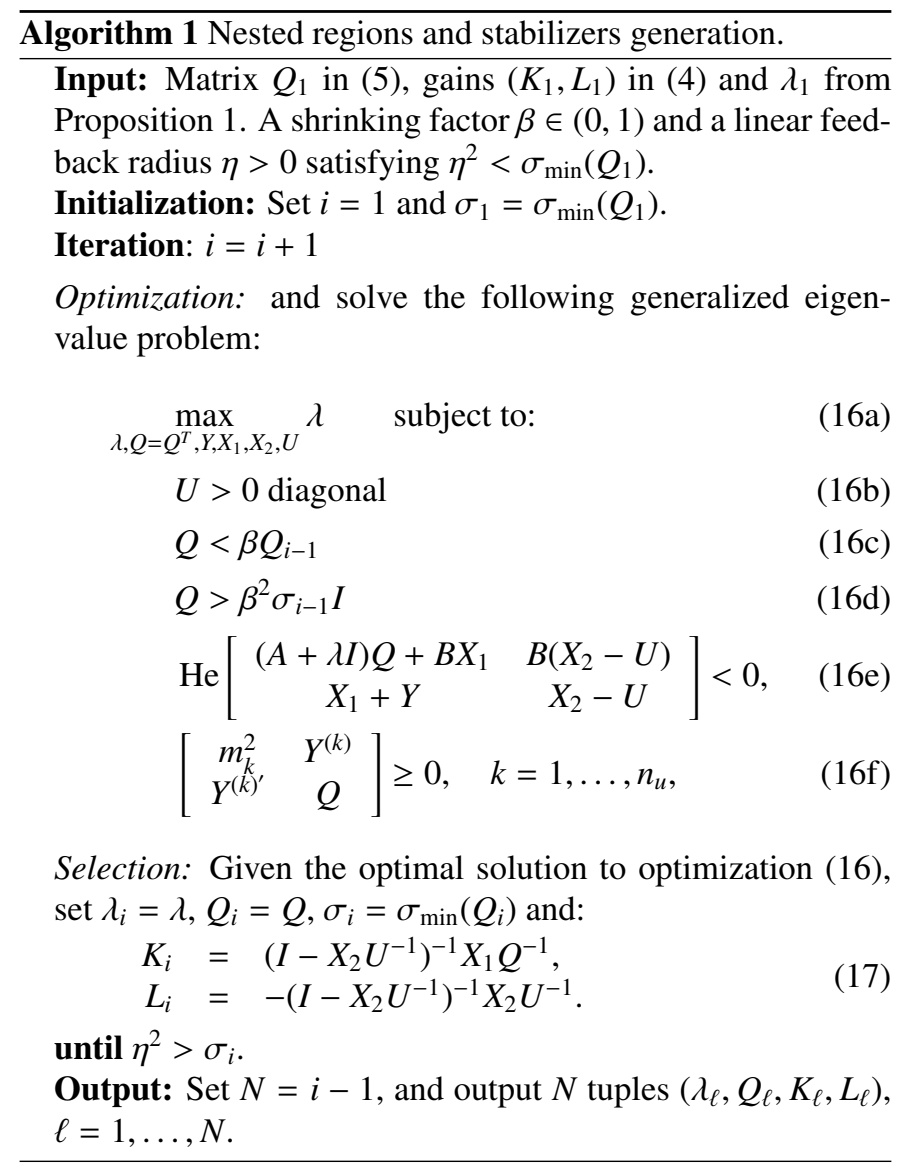

Finally, for a fixed value of $\eta$, integer $N$ is a function of scalar $\beta$ satisfying:

$$
\lim _{\beta \rightarrow 0} N=1, \quad \lim _{\beta \rightarrow 1} N=+\infty .
$$

Proof. From the feasibility conditions of the LMIs established in Proposition 1, it follows that the optimization problem in the algorithm is well-posed, in the sense that it always admits a solution $\lambda_{i} \geq \lambda_{i-1}$. Moreover, the strict inclusion $\mathcal{E}_{i} \subset \mathcal{E}_{i-1}$ is guaranteed by (16c):

$\mathcal{E}_{i}=\left\{x^{T} Q_{i}^{-1} x \leq 1\right\} \subset\left\{x^{T} \beta^{-1} Q_{i-1}^{-1} x \leq 1\right\} \subset\left\{x^{T} Q_{i-1}^{-1} x \leq 1\right\}=\mathcal{E}_{i-1}$.

Conditions (16c)-(16d) ensure that the algorithm terminates after $N$ steps. Indeed one has $\beta^{2} \sigma_{i-1}<\sigma_{i}<\beta \sigma_{i-1}$, and hence

$$
\beta^{2 i-2} \sigma_{1}<\sigma_{i}<\beta^{i-1} \sigma_{1} .
$$

Taking logarithms of both sides of $\eta^{2} \leq \sigma_{N}<\beta^{N-1} \sigma_{1}$, we get that the largest integer $N$ with $\eta^{2} \leq \sigma_{N}$ (which comes from the termination condition) must satisfy the estimate

$$
N<\frac{\log \eta^{2}-\log \sigma_{1}}{\log \beta}+1,
$$

which implies the left limit in (20) because $\eta^{2}<\sigma_{1}$ by definition. Consider now again the termination condition and the left inequality in (21), which yields $\beta^{2((N+1)-1)}<\sigma_{N+1} \leq \eta^{2}$. Taking logarithms again, we get

$$
N>\frac{\log \eta^{2}-\log \sigma_{1}}{2 \log \beta},
$$

which implies the right limit in (20).

Remark 2. In light of Proposition 2, from a practical point of view, Algorithm 1 should be applied by first fixing the local convergence rate $\lambda_{1}$ and solving the LMIs (3) in Proposition 1 with the goal of maximizing the size of $Q_{1}$ (e.g., maximizing its trace). Alternatively, if a certain size $\bar{\alpha}$ of the outer region is required, one may fix $Q_{1} \geq \alpha^{2} I$ and then maximize $\lambda_{1}$ by solving a generalized eigenvalue problem. Then the linearity radius $\eta$ is typically selected as significantly smaller than $\sqrt{\sigma_{\min }\left(Q_{1}\right)}$ (so as to leave a large enough doughnut for the nesting of the ellipsoidal sets before the termination condition), and then the shrinking factor $\beta$ is adjusted by running the algorithm for several values of it with the goal of obtaining a reasonable number $N$ of ellipsoids. In particular, the result (20) clarifies that increasing $\beta$ one expects an increase of $N$, while decreasing $\beta$ one expects a decrease of $N$. A larger $N$ leads to improved performance, due to a more sophisticated control solution, but also increases the computational complexity in terms of the number of gains that need to be stored in the control system.

\section{Hysteresis switching stabilization}

In the previous two sections, a sequence of nested sets $\mathcal{E}_{1} \supset$ $\ldots \supset \mathcal{E}_{N}$ has been determined by way of Algorithm 1, each of them associate with a pair of gains $\left(K_{i}, L_{i}\right)$ and with a guaranteed convergence rate $\lambda_{i} \geq 0$ satisfying the non-decreasing condition (19).

Here, generalizing the approach of [55] and [12], in light of the recent formalism in [17] for the representation of hybrid dynamical systems, we propose a hysteresis switching law that exploits the fact that by construction, for each $q=2, \ldots, N$, set $\mathcal{E}_{q}$ (see the outer bold gray level set in Figure 1 for an example) is a subset of the $\beta$-restriction (see the dashed level set in Figure 1 for an example) of set $\mathcal{E}_{q-1}$ (see the inner bold gray level set in Figure 1 for an example). The intuitive idea behind the hysteresis switching law is to switch to less aggressive control gains (that is, from the gains associated to $\mathcal{E}_{q+1}$ to the gains associated to $\mathcal{E}_{q}$ ) when the state $x$ crosses the boundary of $\mathcal{E}_{q+1}$, but to allow a switch to more aggressive gains (that is, from the gains associated to $\mathcal{E}_{q}$ to the gains associated to $\mathcal{E}_{q+1}$ ) only when the state $x$ crosses the boundary of $\beta \mathcal{E}_{q+1}$; the finite separation between the boundaries of $\mathcal{E}_{q+1}$ and $\beta \mathcal{E}_{q+1}$ provides some level of robustness to noise and prevents chattering problems.

To this aim, we introduce a logic state variable $q \in\{1, \ldots, N\}$ in our control algorithm, and implement a $q$-dependent version of the linear stabilizer (2), as follows:

$$
u=K_{q} x+L_{q} \operatorname{sat}_{m}(u)
$$

where state $q$ evolves according to the following hybrid dynamics:

$$
\begin{array}{ll}
\dot{q}=0, & x \in \mathcal{C}_{q} \\
q^{+}=q+1, & x \in \mathcal{D}_{q}^{+} \\
q^{+}=q-1, & x \in \mathcal{D}_{q}^{-},
\end{array}
$$




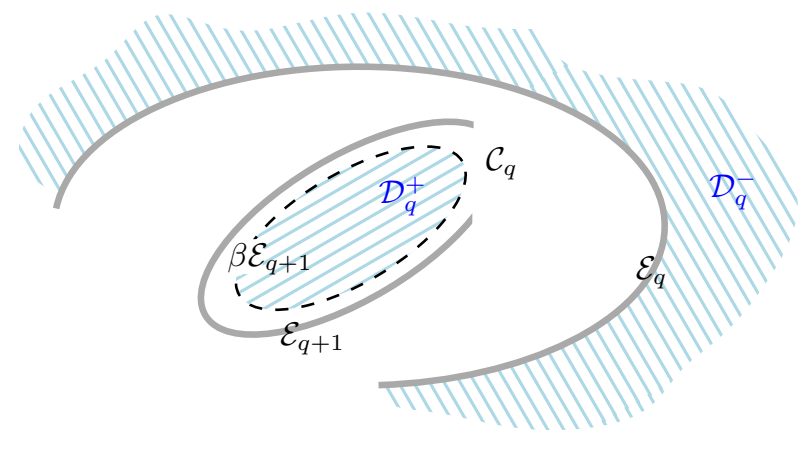

Figure 2: The hysteresis switching mechanism behind control law (22), (23).

where, according to the intuitive representation in Figure 2, increase and decrease of $q$ is enabled, respectively, whenever the state $(x, q)$ belongs to the following sets:

$$
\begin{array}{ll}
\mathcal{D}_{q}^{-}:=\left\{x \in \mathbb{R}^{n}: x^{T} Q_{q}^{-1} x \geq 1\right\}, & q \in\{2, \ldots, N\} \\
\mathcal{D}_{q}^{+}:=\left\{x \in \mathbb{R}^{n}: x^{T} Q_{q+1}^{-1} x \leq \beta\right\}, & q \in\{1, \ldots, N-1\}, \\
\mathcal{D}_{1}^{-}:=\emptyset, \quad \mathcal{D}_{N}^{+}:=\emptyset, &
\end{array}
$$

and the set $C_{q}$ in (22b) is selected as the closure of the set where jumps (or updates) of $q$ are not allowed:

$$
C_{q}=\overline{\mathbb{R}^{n} \backslash\left(\mathcal{D}_{q}^{+} \cup \mathcal{D}_{q}^{-}\right)} .
$$

As customary with the representation in [17] for hybrid systems, the so-called flow set of the hybrid dynamics corresponds to the subset of the state space $\Xi=\mathbb{R}^{n} \times\{1, \ldots, N\}$ where flow of the solutions is allowed, and the so-called jump set of the hybrid dynamics corresponds to the subset of the state space $\Xi$ where jump of the solutions is allowed. Taking a similar approach to [17, eq. (1.10)], they correspond to:

$$
\begin{aligned}
C & =\bigcup_{q \in\{1, \ldots, N\}} C_{q} \times\{q\} \\
\mathcal{D} & =\bigcup_{q \in\{1, \ldots, N\}}\left(\mathcal{D}_{q}^{-} \cup \mathcal{D}_{q}^{+}\right) \times\{q\},
\end{aligned}
$$

and the flow and jump maps are directly derived from (22b) due to the fact that the sets $\mathcal{D}_{q}^{-}, \mathcal{D}_{q}^{+}, q=1, \ldots, N$ are all disjoint.

The next result is the first main theorem of this paper establishing useful properties of the hysteresis switching stabilizer. An appealing feature of this theorem, as compared to previous approaches, such as those in $[55,12]$ is that the well posedness of the hybrid formulation in (22), (23) allows obtaining intrinsic robustness of the stated stability results, by way of the general results in [17, Thm 7.21].

Theorem 1. Consider plant (1), a set of initial parameters $\left(Q_{1}, K_{1}, L_{1}, \lambda_{1}\right)$ coming from Proposition 1 and a family of nested sets $\left(Q_{i}, K_{i}, L_{i}, \lambda_{i}\right), i=1, \ldots, N$ coming from Algorithm 1.

Then the hybrid hysteresis switching state-feedback control law (22), (23) for plant (1) is such that

1. complete solutions exist for all initial conditions, and are all eventually continuous,
2. if parameters $\left(Q_{1}, K_{1}, L_{1}, \lambda_{1}\right)$ came from a solution to (3) of Proposition 1 with $Y=0$, then the origin is globally exponentially stable;

3. the exponential convergence rate increases as the solution approaches the origin. In particular, there exists a scalar $M \geq 1$, such that, for all solutions, the following holds:

$$
x(0,0) \in \beta \mathcal{E}_{k} \quad \Rightarrow \quad|x(t, j)| \leq M \exp \left(-\lambda_{k} t\right)|x(0,0)|,
$$

for all $(t, j) \in \operatorname{dom} x$;

4. the origin is exponentially stable for the closed loop with region of attraction including the set $\mathcal{E}_{1}$.

Proof. First note that dynamics (1), (22), (23) satisfies the hybrid basic conditions in [17, Assumption 6.5] because $C$ and $\mathcal{D}$ are closed, the flow map is continuous and the jump map can be continuously extended. Then existence and completeness of maximal solutions are guaranteed by [17, Proposition 6.10] and the well posedness of (1), (2) established in Proposition 1 (guaranteeing that item (b) of Proposition 6.10 in [17] never occurs), together with the fact that item (c) of Proposition 6.10 never occurs because $C \cup \mathcal{D}=\mathbb{R}^{n} \times\{1, \ldots, N\}$. The fact that all solutions are eventually continuous is proved below.

Consider now any solution $\xi=(x, q)$ to $(1),(22),(23)$. Let $\xi(0,0)=\left(x_{0}, q_{0}\right)$ and denote by $\mathcal{R}_{1}$ the region of attraction of the closed-loop system with $(K, L)=\left(K_{1}, L_{1}\right)$ which, by construction, contains the ellipsoid $\mathcal{E}_{1}$ (see Proposition 1). Two cases can be considered

a) $x_{0} \in C_{q_{0}}$

b) $x_{0} \notin C_{q_{0}}$

In case a), the initial state belongs to the set $\mathcal{E}_{q_{0}}$ which is forward invariant for the closed-loop dynamics with gains $(K, L)=$ $\left(K_{q_{0}}, L_{q_{0}}\right)$ (again from Proposition 1$)$. As a consequence, introducing the coefficients

$$
c_{i}:=\sqrt{\frac{\lambda_{\max }\left(Q_{i}\right)}{\lambda_{\min }\left(Q_{i}\right)}} \geq 1 \forall i=1, \ldots, N,
$$

the following exponential estimate is in force

$$
|x(t, 0)| \leq c_{q_{0}} \exp \left(-\lambda_{q_{0}} t\right)|x(0,0)|
$$

for any $t \geq 0$ such that $(t, 0) \in \operatorname{dom} \xi$ (because this means that the solution never jumps thereby remaining in $C_{q_{0}}$ ). If the supremum of such $t$ is $\infty$, then bound (25) proves the claim. Otherwise, the solution reaches the boundary $\partial\left(\beta \mathcal{E}_{q_{0}+1}\right)$ at some finite time $\left(t_{1}, 0\right) \in \operatorname{dom} \xi$ such that $\left(t_{1}, 1\right) \in \operatorname{dom} \xi$ and $q\left(t_{1}, 1\right)=q_{0}+1, x\left(t_{1}, 1\right) \in \partial\left(\beta \mathcal{E}_{q_{0}+1}\right) \subset \mathcal{E}_{q_{0}+1}$ because $q$ jumps from $q\left(t_{1}, 0\right)=q_{0}$ to $q\left(t_{1}, 1\right)=q_{0}+1$.

Since $\mathcal{E}_{q_{0}+1}$ is forward invariant for continuous dynamics with gains $(K, L)=\left(K_{q_{0}+1}, L_{q_{0}+1}\right)$, one has

$$
|x(t, 1)| \leq c_{q_{0}+1} \exp \left(-\lambda_{q_{0}+1}\left(t-t_{1}\right)\right)\left|x\left(t_{1}, 0\right)\right|
$$

for any $t \geq t_{1}$ satisfying $(t, 1) \in \operatorname{dom} \xi$, and thus, incorporating (25) and using (19),

$$
|x(t, j)| \leq c_{q_{0}+1} c_{q_{0}} \exp \left(-\lambda_{q_{0}} t\right)|x(0,0)|
$$


for any $t \geq 0$ such that $x(t, j) \in \operatorname{dom} \xi$ with $j \in\{0,1\}$.

The above argument can be repeated $\ell \leq N-1$ times, thus proving items (2) and (3) of the theorem. Moreover, note that all selections of $K_{q}$ and $L_{q}$ exponentially stabilize the origin. Then all solutions evolving in $\mathcal{E}_{q}, q<N$, eventually reach the neighborhood of the origin $\beta \mathcal{E}_{q}$ and jump, eventually reaching $\beta \mathcal{E}_{N-1} \subset$ int $\mathcal{E}_{N}$. From there the evolution is continuous, thus proving item (1). Summarizing, it has been shown that

$$
|x(t, j)| \leq M \exp \left(-\lambda_{q_{0}} t\right)|x(0,0)| \forall t \geq 0, j=0,1, \ldots, \ell,
$$

where the constant $M$ is given by

$$
M=\Pi_{i=1}^{N} c_{i} .
$$

Let us consider now case b). If $x(0,0) \in \mathcal{E}_{\bar{q}}$ for some $\bar{q} \in$ $\{1, \ldots, N\}$, the jump map (22b) guarantees that after, $\ell \leq \mid q_{0}-$ $\bar{q} \mid \leq N-1$ jumps, the hybrid state $\xi(0, \ell)$ is such that

$$
x(0, \ell) \in C_{\bar{q}}, \quad q(0, \ell)=\bar{q} .
$$

Therefore one turns back to the scenario of case a), with $x(0,0)$ replaced by $x(0, \ell)$. This shows that (24) is fulfilled with $M$ given by (28). We point out that clearly it is possible to find some sharper estimates on the bound $M$.

The only case that has not been addressed yet is $x(0,0) \notin \mathcal{E}_{1}$, which is considered in item (4) for the case where $\left(K_{1}, L_{1}\right)$ are globally exponentially stabilizing. In this case, it is trivial to see that the solution performs a finite number of jumps until $q$ becomes 1. From there, Proposition 1 gives an exponential bound until $x \in \partial\left(\beta \mathcal{E}_{1}\right)$, and then the analysis above applies. $\diamond$

Remark 3. (Global, semi-global and regional results) Theorem 1 (as well as Theorem 2 given in the next section) guarantees different stability properties on the closed loop, depending on how the family of solutions to (3) were determined. In particular, according to the results of Proposition 1 (applied with $\lambda=0$ ), for exponentially stable plants it will be possible to choose the 1-st solution in such a way that the corresponding gains are globally exponentially stabilizing (namely with $Y=0$ ), so that global exponential stability will hold for the hysteresis switching feedback scheme. Alternatively, for marginally stable/unstable plants it is possible to achieve semiglobal exponential stability by choosing the largest invariant set (corresponding to the 1-st solution) arbitrarily large. Finally, for exponentially unstable plants, it will only be possible to achieve regional exponential stability, possibly maximizing the size of the largest invariant set $\mathcal{E}_{1}$ : indeed, it is well known (see, e.g., [34]) that exponentially unstable plants with bounded control inputs have null controllability regions that are bounded in the exponentially unstable directions, so that they cannot be globally nor semiglobally stabilized.

\section{Scheduled stabilization}

As opposed to the switching solution proposed in the previous section, we now use a different strategy for exploiting the nested family of stabilizers determined in Section 2.2, which does not require any switching (thus avoiding discontinuities in the control input). This solution is based on interpolating the control law as suggested in $[11,13]$, which is resemblant of the techniques in $[27,29]$. To this aim, the integer logic variable $q$ of the previous section is here converted into a scheduling parameter belonging to the real interval $[1, N]$.

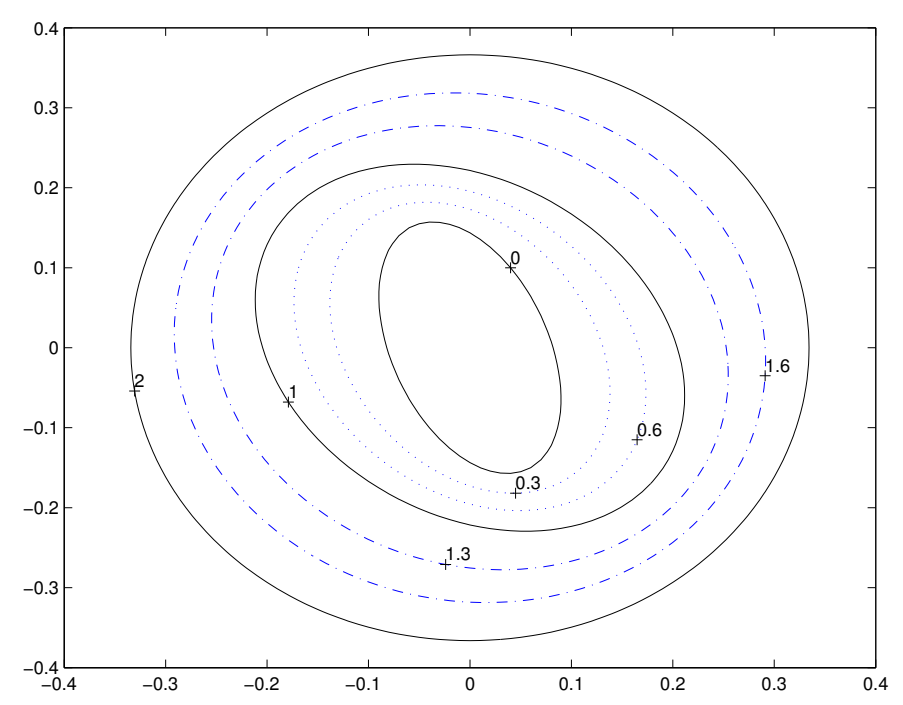

Figure 3: The nested sets and their interpolation using the parameter $\alpha$.

In particular, the matrices $X_{1, i}, X_{2, i}$ and $Q_{i}, i=1, \ldots, N$ obtained from Algorithm 1 can be used to define a Lipschitz nonlinear control law as follows. Let

$$
\begin{aligned}
& \lfloor q\rfloor:=\max \{1, \text { floor }(q)\} \\
& \lceil q\rceil:=\min \{N, \operatorname{ceil}(q)\},
\end{aligned}
$$

(so that for $q \in[1, N],\lceil q\rceil$ denotes the smallest integer larger than $q$, whereas $\lfloor q\rfloor$ denotes the largest integer smaller than $q$ ), and define interpolated variables through convex combination:

$$
\begin{aligned}
Q(q) & =(\lceil q\rceil-q) Q_{\lfloor q\rfloor}+(q-\lfloor q\rfloor) Q_{\lceil q\rceil} \\
U(q) & =(\lceil q\rceil-q) U_{\lfloor q\rfloor}+(q-\lfloor q\rfloor) U_{\lceil q\rceil} \\
X_{1}(q) & =(\lceil q\rceil-q) X_{1\lfloor q\rfloor}+(q-\lfloor q\rfloor) X_{1\lceil q\rceil} \\
X_{2}(q) & =(\lceil q\rceil-q) X_{2\lfloor q\rfloor}+(q-\lfloor q\rfloor) X_{2\lceil q\rceil} .
\end{aligned}
$$

Then we may use the real parameters $q \in[1, N]$ (whose selection is specified below), to define an implicit, nonlinear statefeedback control law

$$
u=K_{s}(q(x)) x+L_{s}(q(x)) \operatorname{sat}_{m}(u)
$$

where subscript "s" stands for "scheduled" and we define, similar to (4),

$$
\begin{aligned}
& K_{s}(q)=\left(I-X_{2}(q) U(q)^{-1}\right) X_{1}(q) Q(q)^{-1} \\
& L_{s}(q)=-\left(I-X_{2}(q) U(q)^{-1}\right) X_{2}(q) U(q)^{-1} .
\end{aligned}
$$

Finally, the function $x \mapsto q(x)$ is selected as follows:

$$
q(x)= \begin{cases}1 & \text { if } x \notin \mathcal{E}_{1} \\ N & \text { if } x \in \mathcal{E}_{N} \\ q^{*}(x) & \text { if } x \in \mathcal{E}_{1} \backslash \mathcal{E}_{N},\end{cases}
$$


where $x \mapsto q^{*}(x)$ is the unique solution to the following implicit equation, whose existence and properties are guaranteed by the next lemma (whose proof is given in the appendix):

$$
x^{T} Q^{-1}\left(q^{*}(x)\right) x=1, \quad x \in \mathcal{E}_{1} \backslash \mathcal{E}_{N} .
$$

Lemma 3. Equation (33b) implicitly defines a unique, bounded and Lipschitz continuous function $q^{*}(x)$ in $\mathcal{E}_{1} \backslash \mathcal{E}_{N}$.

We are now ready to state our second main result, establishing desirable properties of the Lipschitz nonlinear scheduled stabilizer.

Theorem 2. Consider plant (1), a set of initial parameters $\left(Q_{1}, X_{11}, X_{21}, U_{1}, \lambda_{1}\right)$ coming from Proposition 1 and a family of nested sets $\left(Q_{i}, X_{1 i}, X_{2 i}, U_{i}, \lambda_{i}\right), i=2, \ldots, N$ coming from $A l$ gorithm 1.

Then the Lipschitz nonlinear state-feedback control law (30)-(33) is such that:

1. the origin is exponentially stable for the closed loop with region of attraction including the set $\mathcal{E}_{1}$;

2. if parameters $\left(Q_{1}, X_{1}, X_{2}, U_{1}, \lambda_{1}\right)$ came from a solution to (3) of Proposition 1 with $Y=0$, then the origin is globally exponentially stable;

3. the exponential convergence rate increases as the solution approaches the origin. In particular, there exists a positive scalar M such that, for all solutions, the following holds:

$$
x(0) \in \mathcal{E}_{k} \quad \Rightarrow \quad|x(t)| \leq M \exp \left(-\lambda_{k} t\right)|x(0)|
$$

for all $t \geq 0$.

Proof. Consider the Lipschitz Lyapunov function candidate

$$
V(x, q(x)):=\tilde{V}(x, q(x))+N-q(x)
$$

where $\tilde{V}(x, q(x))=x^{T} Q^{-1}(q(x)) x$ and $q(x)$ is assigned by (33) and is Lipschitz thanks to Lemma 3. From (33), the scheduling parameter $q(x)$ is constant in $\mathcal{E}_{1}^{c}:=\mathbb{R}^{n} \backslash \mathcal{E}_{1}$ and in $\mathcal{E}_{N}$, where it is equal to 1 and $N$ respectively. Hence one has

$$
\dot{V}(x, q(x))=\dot{\tilde{V}}(x, q(x))
$$

for almost all $x \in \mathcal{E}_{1}^{c} \cup \mathcal{E}_{N}$. Moreover, following the derivations leading to (12), there exists $\gamma>0$ satisfying for almost all $x \in$ $\mathcal{E}_{N}$

$$
\dot{V}(x, q(x))=\dot{\tilde{V}}(x, q(x)) \leq-2 \gamma \tilde{V}(x, q(x)) .
$$

On the other hand, from (33b) the identity

$$
\tilde{V}(x, q(x))=\tilde{V}\left(x, q^{*}(x)\right)=1
$$

holds whenever $x \in \mathcal{E}_{1} \backslash \mathcal{E}_{N}$, and therefore, if the state $x$ belongs to such set, the derivative of $V(x, q(x))$ reduces to $\dot{V}(x, q(x))=$ $-\dot{q}(x)=-\dot{q}^{*}(x)$, where we also used the definition in (33).

Differentiating both sides of (36), we obtain $\nabla_{x} \tilde{V}\left(x, q^{*}(x)\right)=0$, which can be developed to get

$$
\dot{q}^{*}(x)=-\left(\left.x^{T} \frac{\partial Q^{-1}(q)}{\partial q}\right|_{q=q^{*}(x)} x\right)^{-1} 2 x^{T} Q^{-1}\left(q^{*}(x)\right) \dot{x} .
$$

As a consequence we obtain:

$$
\dot{V}(x, q(x))=-\dot{q}^{*}(x)=\frac{2 x^{T} Q^{-1}\left(q^{*}(x)\right)\left(A x+B \operatorname{sat}_{m}(u)\right)}{x^{T} M\left(q^{*}(x)\right) x},
$$

where for almost all $q \in[1, N]$ (namely for all non-integer values of $q$ in that interval) ${ }^{2}$

$$
M(q):=Q^{-1}(q)\left(Q_{\lfloor q\rfloor}-Q_{\lceil q\rceil}\right) Q^{-1}(q)>0 .
$$

Due to convexity, LMIs (3) are still satisfied by the interpolated variables (30), hence, selecting the control input as the scheduled feedback (31) and adapting the proof of item (2) in Proposition 1 to bound the numerator in (37), we obtain for some scalar $\tilde{\gamma}>0$

$$
\dot{V}(x, q(x)) \leq-2 \frac{\tilde{\gamma}}{\tilde{M}} \tilde{V}(x, q(x))
$$

for almost all $x \in \mathcal{E}_{1} \backslash \mathcal{E}_{N}$, where

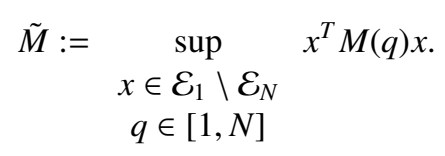

The existence of a uniformly negative upper bound for $\dot{V}(x, q(x))$ for almost all $x \in \mathcal{E}_{1}$ implies exponential stability of the origin with region of attraction containing $\mathcal{E}_{1}$, by following the derivations in [37, page 99], thus proving item (1).

Item (2) follows very similar steps. Indeed, from item (1) of Proposition 1, we have that bound (35) also holds for almost all $x \in \mathcal{E}_{1}^{c}$, and therefore the negative upper bound for $\dot{V}(x, q(x))$ holds almost globally. The argument in [37] then implies global exponential stability of the origin. Consider now item (3). To prove the increasing behavior of convergence rate one can make the preliminary observation that, given the sequence of solutions $\left(Q_{i}, X_{1 i}, X_{2 i}, U_{i}, \lambda_{i}\right)$ of (16) for $i=1, \ldots, N$ coming from Algorithm 1, inequality (16e) with $Q=Q_{i}$ is still satisfied by any $\lambda_{k}$ with $i \geq k \geq 1$. By convexity, the same feature is transferred to the interpolated variables (30), i.e.

$$
\operatorname{He}\left[\begin{array}{cc}
\left(A+\lambda_{k} I\right) Q(q)+B X_{1}(q) & B\left(X_{2}(q)-U(q)\right) \\
X_{1}(q)+Y & X_{2}(q)-U(q)
\end{array}\right]<0
$$

for any $q \geq k \geq 1$. On the other hand, the function $q^{*}(x)$ defining the scheduled parameter has been proven to be nondecreasing along the solution (see conditions (37) and (39)). This property together with inequality (40) guarantees that, if the initial state $x(0)$ belongs to the ellipsoid $\mathcal{E}_{k}$ for some $k \in\{1, \ldots, N\}$, then the convergence rate of the solution $x(t)$ is not smaller than $\lambda_{k}$ by condition (19), thus also proving item (3).

\footnotetext{
${ }^{2}$ One clearly sees that $M(q)=\frac{d Q^{-1}(q)}{d q}$ : this identity follows from (30) and keeping in mind that for a matrix function $q \mapsto P(q)$, we have that $P(q) P^{-1}(q)=$ 1, implies $\frac{\partial P(q)}{\partial q}=-P(q) \frac{\partial P^{-1}(q)}{\partial q} P(q)$. See the proof of Lemma 3 for further details about identity (37) and positive definiteness of $M(q)$.
} 
Remark 4. (Computation of the scheduling parameter $q^{*}$ ) Since the algebraic solution of equation (33b) (namely, the function $x \mapsto q^{*}(x)$ ) does not appear to be easy to find, ${ }^{3}$ a dynamic selection for $q$ can be employed, which integrates the plant dynamics to asymptotically estimate the correct value of $q^{*}$. While this can be done $e$.g. by bisection methods, in $[11,13]$ a dynamical approach was suggested, which is based on suitably integrating the dynamics of $q^{*}(x)$ implicitly defined by condition (33b). In particular, condition (33b) can be first derived with respect to time, to obtain

$$
\frac{d}{d t} x^{T} Q^{-1}(q) x=2 x^{T} Q^{-1}(q) \dot{x}+\left(x^{T} \frac{d Q^{-1}(q)}{d q} x\right) \dot{q}=0
$$

from which it is possible to compute $\dot{q}$ and write a dynamic equation governing a new state $q$. In particular, taking $M(q)$ as in (38) and defining

$$
g(x, q):=\ell\left(x^{T} Q^{-1}(q) x-1\right),
$$

a robust implementation of dynamics $\dot{q}$ with feedback injection $g(x, q)$, where scalar $\ell>0$ is arbitrary and may be tuned for optimized performance, is given by

$$
\dot{q}= \begin{cases}\max \{0,-g(x, q)\}, & \text { if } q \leq 1 \\ \frac{2 x^{T} Q^{-1}(q)\left(A x+B \operatorname{sat}_{m}(u)\right)}{x^{T} M(q) x}-g(x, q), & \text { if } q \in(1, N) \\ \min \{0,-g(x, q)\}, & \text { if } q \geq N,\end{cases}
$$

with initial state $q(0) \in[1, N]$. It can be shown that, under the stated assumptions, the right hand side of (41) is bounded and well defined almost everywhere, and that the interval $[1, N]$ is forward invariant for $q$ (that is, the state $q$ never leaves such interval). In fact, a more precise description of the $q$ dynamics would require the use of differential inclusions because $Q(\cdot)$ in (30) is Lipschitz so that its generalized derivative should be used in place of matrix $M$. To keep the discussion simple, we abuse notation and treat the $q$ dynamics as a differential equation. To better appreciate how the algorithm works in practice, few more comments are in order.

First, the computations in (41) require the knowledge of the state $x$, which might appear limiting; however, in the antiwindup application such state $x$ is exactly the state of the antiwindup compensator, and then it is explicitly available.

Next, the role of the terms appearing in the right hand side of (41) can be explained as follows. In all cases, the term $-g(x, q)$ has the role of correcting a "wrong" initialization of the state $q$ in (41), since the initial value of $q(0)$ usually does not satisfy the condition $x^{T}(0) Q^{-1}(q(0)) x(0)=1$ (the "correct" initialization would require the solution of (33b) to be already known); the term $-g(x, q)$ is a stabilizing feedback which disappears as $x^{T} Q^{-1}(q) x$ gets closer and closer to 1 . For the case $q \in(1, N)$,

\footnotetext{
${ }^{3}$ Several solutions have been proposed to determine $\alpha$ by on line search methods and other computationally intensive approaches; see e.g. [30, 27] and references therein.
}

the other term appearing in the right hand side of (41) is exactly the dynamics that, according to equation $(*)$ above, ensures forward invariance of the set $\left\{(x, q): x^{T} Q^{-1}(q) x=1\right\}$, namely, it ensures that if $x^{T} Q^{-1}(q) x=1$ at some time $t_{0}$, then the motion of $q$ is suitably coordinated with the motion of $x$ so to keep the relation $x^{T} Q^{-1}(q) x=1$ satisfied also for all $t \geq t_{0}$. Now, recalling that by (29) the value of $q$ is assumed to range in the interval $[1, N]$, the max operator is used in the case $q \leq 1$ to avoid that the value of $q$ decreases below 1 once it has reached such a lower bound, and similarly the min operator is used in the case $q \geq N$ to avoid that the value of $q$ increases above $N$ (as mentioned, the discontinuity introduced by such terms can be formally dealt with by using differential inclusions). Note that, although the set $[1, N]$ can be formally shown to be forward invariant under (41), allowing $q<1$ or $q>N$ in the right hand side of (41) ensures robustness to numerical errors.

Finally, from the above discussion it is clear that the role of the parameter $\ell$ in $g(x, q)$ is to make the feedback action exerted by $g(x, q)$ in (41) more or less aggressive, thus influencing how fast the condition $x^{T} Q^{-1}(q) x=1$ is achieved. Hence, in practice the value of $\ell$ should be chosen large enough to ensure that $q$ quickly becomes a good solution of $x^{T} Q^{-1}(q) x=1$; practical limits on how large the value of $\ell$ can be taken are clearly given by the possible numerical integration problems that might arise due to excessively large values. Note that, since $\ell$ only affects a fast initial transient, its effect on the performance of the antiwindup compensation are quite negligible.

\section{Application to nonlinear model recovery anti-windup}

In this section, similar to $[12,11,13]$, and following the paradigm in [42] and [57, Part II], we apply the proposed constructions to model recovery anti-windup design, which is capable of transforming a stability and performance recovery problem into a stabilization problem (applied to the mismatch dynamics). To this end, we consider a linear plant given by:

$$
\mathcal{P}\left\{\begin{aligned}
\dot{x}_{p} & =A x_{p}+B u_{p}+B_{w} w \\
y & =C_{y} x_{p}+D_{y} u_{p}+D_{y w} w \\
z & =C_{z} x_{p}+D_{z} u_{p}+D_{z w} w
\end{aligned}\right.
$$

where $x_{p} \in \mathbb{R}^{n_{p}}$ is the plant state, $u_{p} \in \mathbb{R}^{n_{u}}$ is the control input, $w \in \mathbb{R}^{n_{w}}$ is an exogenous input (possibly containing disturbances, references and measurement noise), $y \in \mathbb{R}^{n_{y}}$ is the measurement output and $z \in \mathbb{R}^{n_{z}}$ is the performance output.

As customary in the anti-windup setting, we assume that a (linear) controller ${ }^{4}$ has been designed to induce desirable performance when interconnected to the plant without saturation:

$$
C\left\{\begin{array}{l}
\dot{x}_{c}=A_{c} x_{c}+B_{c u} u_{c}+B_{c w} w \\
y_{c}=C_{c} x_{c}+D_{c u} u_{c}+D_{c w} w
\end{array}\right.
$$

where $x_{c} \in \mathbb{R}^{n_{c}}$ is the controller state and $y_{c} \in \mathbb{R}^{n_{u}}$ is the controller output.

\footnotetext{
${ }^{4}$ We assume here that controller (43) be linear but our results extend straightforwardly to the case of nonlinear controllers.
} 
In the case without input saturation, we call unconstrained closed-loop system the direct feedback interconnection between the controller (43) and the plant (42) via the equations

$$
u=y_{c}, \quad u_{c}=y \text {. }
$$

A necessary assumption for anti-windup design is that the unconstrained closed-loop system (42), (43), (44) is well posed and internally stable.

The so-called saturated closed-loop system corresponds to the interconnection between (42), (43) and the level $\bar{u} \in \mathbb{R}_{>0}^{n_{u}}$ saturated interconnection

$$
u=\operatorname{sat}_{\bar{u}}\left(y_{c}\right), \quad u_{c}=y
$$

where each element of $\bar{u}$ indicates the (symmetric) saturation level of the corresponding input channel.

The MRAW compensation scheme first proposed in [42], later revisited in [54] (therein it was called " $\mathcal{L}_{2}$ anti-windup") and finally well surveyed in [57, Part II], corresponds to inserting the following anti-windup filter, or mismatch dynamics, in the closed loop:

$$
\mathcal{A W}\left\{\begin{array}{l}
\dot{x}_{a w}=A x_{a w}+B\left(\operatorname{sat}_{m}\left(y_{c}+v\right)-y_{c}\right), \\
y_{a w}=C_{y} x_{a w}+D_{y}\left(\operatorname{sat}_{m}\left(y_{c}+v\right)-y_{c}\right),
\end{array}\right.
$$

with $x_{a w}(0)=0$ and where $v$ is a signal to be designed. The saturation level $m$ is defined as

$$
m:=(1-\varepsilon) \bar{u}
$$

where $\varepsilon \in(0,1)$ is a given arbitrarily small constant, so that $\mathrm{sat}_{m}$ corresponds to a restricted saturation with respect to $\mathrm{sat}_{\bar{u}}$. The restriction of the saturation level by an (arbitrarily small) quantity $\varepsilon$ is actually a necessary condition for stabilization and is therefore key to being able to prove Lemma 4 below. See [42, Remark 2.2] for an example where this assumption is necessary. Note that this may appear somewhat close in spirit to the recent works on anticipatory anti-windup designs (see, e.g., [51]), but its spirit is actually different because one typically selects $\varepsilon$ as a small positive scalar.

The interconnection of (46) to the closed loop system (42), (43) via the anti-windup interconnection

$$
u_{c}=y-y_{a w}, \quad u=\operatorname{sat}_{m}\left(y_{c}+v\right)
$$

yields the so called anti-windup closed-loop system, shown in Fig. 4.

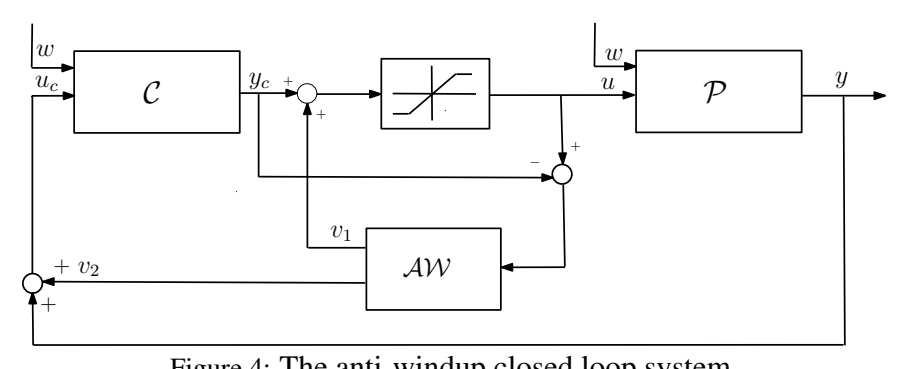

Figure 4: The anti-windup closed loop system.
In $[42,54]$ the anti-windup design goals were formally stated as follows. Denote by $u_{l}, z_{l}$ the linear controller output and the plant performance output of the unconstrained closed-loop system and by $u_{a}, z_{a}$ the corresponding signals of the anti-windup closed-loop system. Then the signal $v$ should be selected in such a way that the following two properties hold:

1 . if $u_{l}$ never exceeds the saturation bounds $\eta$, then $z_{a}$ coincides with $z_{l}$ (shortly, $u_{l} \equiv \operatorname{sat}_{\eta}\left(u_{l}\right) \Longrightarrow z_{a} \equiv z_{l}$ );

2. if $\mathrm{dz}_{m}\left(u_{l}\right)$ (i.e. $u_{l}-\mathrm{sat}_{m}\left(u_{l}\right)$, the portion of the control input in the linear closed loop exceeding the restricted saturation bounds ${ }^{5} \mathrm{~m}$ ) is an $\mathcal{L}_{2}$ signal then the difference between $z_{a}$ and $z_{l}$ is an $\mathcal{L}_{2}$ signal (shortly, $\mathrm{dz}_{m}\left(u_{l}\right) \in \mathcal{L}_{2} \Longrightarrow$ $\left.\left(z_{a}-z_{l}\right) \in \mathcal{L}_{2}\right)$.

In the past decade, several papers have been published describing different selections for the signal $v$ inducing various levels of stability and performance on the anti-windup closedloop system. In particular, the first technique proposed in [42] corresponded to selecting $v=k\left(x_{a w}\right)$ as a static nonlinear function of the anti-windup compensator state. In the special case where the plant was not unstable, this selection was linear and corresponded to $v=K x_{a w}$ with $K$ selected based on suitable passivity properties to ensure stability (note that this selections had interesting connections with the scheme proposed in [49]). Later on, in [54], which dealt with exponentially stable plants, for performance improvement this selection was chosen as $v=K x_{a w}+L\left(\operatorname{sat}_{m}\left(y_{c}+v\right)-y_{c}\right)$, thus introducing a nonlinear algebraic loop in the scheme, and determining $L$ so that this loop was well posed (this improved selection has interesting connections with the work in [23]). Nonlinear selections of the signal $v$ within the MRAW framework were also proposed later. In particular, in [4], $v$ was a sampled data signal chosen as a piecewise affine function of the state $x_{a w}$ by relying on explicit solutions to the discrete-time Receding Horizon Control (RHC) strategy. In [39] and [15], a nonlinear function also involving measurements of some plant states was used to obtain large operating regions in the presence of exponentially unstable modes in the plant dynamics. Finally, in [55], a hysteresis switching approach was proposed to enforce improved transients when the plant is exponentially stable. This follow-up approaches are those of $[12,13,11]$, partly reorganized and presented in this paper.

The following lemma shows that the stability properties of the anti-windup closed-loop are equivalent to the stability properties of a simpler, input saturated system.

Lemma 4. Given $m$ as in (47), if the system

$$
\dot{x}_{a w}=A x_{a w}+B \operatorname{sat}_{m}(v)
$$

with the (nonlinear and implicit) selection

$$
v=f\left(x_{a w}, \operatorname{sat}_{m}(v)\right)
$$

is well posed, has a globally Lipschitz right hand side and is globally (respectively, regionally) asymptotically stable, then

\footnotetext{
${ }^{5}$ As shown in [42], it is necessary to consider such a restriction to correctly characterize the marginally stable/unstable case.
} 
the anti-windup closed-loop system (46), (42), (43), (48) is well-posed and globally (respectively, regionally) asymptotically stable.

Proof. The proof is similar to the proofs in [42] and [54], and is based on the fact that rewriting the anti-windup closed-loop system dynamics (46), (42), (43), (48) in the coordinates $\left(x-x_{a w}, x_{c}, x_{a w}\right)$, a cascade structure is revealed where a first subsystem (with state $\left(x-x_{a w}, x_{c}\right)$ ) reproducing the linear unconstrained closed-loop system drives the anti-windup compensator dynamics (46), (50), via the controller output $y_{c}$. This second subsystem (which may be nonlinear due to the nonlinear nature of $v_{1}$ in (50)) can be written as in (49) with a disturbance signal whose norm can be bounded by a constant times $\left\|\mathrm{dz}_{\eta}\left(y_{c}\right)\right\|$ (see [42] for details). Global (respectively, regional) stability of the cascaded system then follows from the forward completeness of each subsystem which, in turns, derives from the global Lipschitz property of the right hand side. $\diamond$

Corollary 1. Selecting the auxiliary input $v$ as the solution of one of the algebraic loops

$$
\begin{aligned}
& v=K_{q} x_{a w}+L_{q} \operatorname{sat}_{m}(v) \\
& v=K_{s}\left(q\left(x_{a w}\right)\right) x_{a w}+L_{s}\left(q\left(x_{a w}\right)\right) \operatorname{sat}_{m}(v)
\end{aligned}
$$

with gains assigned either by the hysteresis switching scheme as in (22)-(23) or by the scheduled scheme as in (31)-(32)-(33), the anti-windup closed-loop system (46), (42), (43), (48) is guaranteed to be well-posed and regionally asymptotically stable. Moreover, if the open-loop plant is exponentially stable, then the anti-windup closed-loop system is globally asymptotically stable.

Remark 5. The performance improvement guaranteed by the proposed design of $v$ in Corollary 1 corresponds to establishing suitable increasingly fast convergence rates of $x_{a w}$ to zero, that can be established because the results of Theorems 1 and 2 carry over to the anti-windup dynamics. Following the derivations in [42] and the later work [54], it is possible indeed to relate the anti-windup state $x_{a w}$ to the mismatch $x-x_{l}$ between the actual plant response and the "unconstrained" response that would have been experienced in the absence of saturation (the information about this unconstrained response is actually stored in the anti-windup dynamics (46)). While it is beyond the scope of this paper to precisely characterize such a performance improvement, it is worth mentioning that proceeding in similar ways to $[42,54,55]$ (see also [57, Part 3]) it is possible to relate the estimate of the domain of attraction provided by Theorems 1 and 2 to the energy spent by the unconstrained input $u_{l}$ outside the restricted saturation bounds (i.e., the $\mathcal{L}_{2}$ norm of $u_{l}-\operatorname{sat}\left(u_{l}\right)$ ). This characterization is beyond the scope of this paper, which instead is mainly focused on the design of the stabilizer $v$ and its qualitative properties.

\section{Examples}

\subsection{Mass-spring-damper system}

We consider the same exponentially stable example used in [12], i.e. a mass-spring system whose equations of motion are

$$
\begin{gathered}
\dot{x}=\left[\begin{array}{cc}
1 & 0 \\
-k / m & -f / m
\end{array}\right] x+\left[\begin{array}{c}
0 \\
1 / m
\end{array}\right] \operatorname{sat}_{1}(u) \\
y=z=\left[\begin{array}{ll}
1 & 0
\end{array}\right] x
\end{gathered}
$$

where $x=(\xi, \dot{\xi})$ are the position and speed of the body of mass $m=1$, and the elastic and damping coefficients are $k=1$ and $f=0.005$. The a priori given unconstrained controller in [54] is $y_{c}=C_{f b}(s)\left(C_{f f}(s) r-u_{c}\right)$ with $C_{f f}(s)=\frac{5}{2 s+5}$, $C_{f b}=500 \frac{(s+15)^{2}}{s(s+80)}$. We follow the strategy suggested in Remark 2 , we fix $\lambda_{1}=1.2670$ and then select the linear feedback radius $\eta=0.01$. Setting the shrinking factor $\beta=0.6$, a family of $N=11$ nested ellipsoids has been generated using Algorithm 1, with associated exponential convergence rates $\lambda_{i}$ ranging from $\lambda_{1}=1.2670$ to $\lambda_{N}=13.6663$. The construction of ellipsoids has been performed in Matlab using the solver SeDuMi, and the total elapsed time of computation was $17 \mathrm{sec}-$ onds. Switched and scheduled anti-windup compensators have been implemented as illustrated in Corollary 1. For the scheduled solution, the value $\ell=60$ has been used. As a comparison, an $\mathcal{L}_{2}$ anti-windup compensator has also been considered using the gains $K_{1}, L_{1}$ given by the algorithm. Figure 5 reports the arising responses. The switched and the scheduled constructions significantly outperform the linear anti-windup scheme. Moreover, from the middle plot it can be appreciated that the scheduled strategy reduces the unpleasant discontinuities at the plant input which characterize the switching technique. The lower plot compares the switching signal to the scheduling signal.

\subsection{TAFA system}

The example of an exponentially unstable system is also considered to further validate the proposed constructions. The linearized model of the short period longitudinal dynamics of TAFA (Tailless Advanced Fighter Aircraft) [3] is given by

$$
\begin{aligned}
& \dot{x}=A x+B \operatorname{sat}_{m_{v}}(u), \\
& y=z, \quad z=\left[\begin{array}{ll}
0 & 1
\end{array}\right] x,
\end{aligned}
$$

where

$$
A=\left[\begin{array}{cc}
-0.9 & 1.0 \\
5.9375 & -2.1
\end{array}\right], \quad B=\left[\begin{array}{l}
0 \\
8
\end{array}\right] .
$$

The state $x=(\alpha, \delta)$ coincides with the measured output, and the saturation level is $m_{v}=0.35 \mathrm{rad}$. For a given reference signal $r \in \mathbb{R}$, the unconstrained controller is assigned by

$$
y_{c}=C_{f b}(s)\left(C_{f f}(s) r-\delta\right)+K_{s} x,
$$

with $C_{f f}(s)=\frac{1.4 s+1}{1.5 s+1}, C_{f b(s)}=\frac{1.5 s+1}{8 s}, K_{s}=-\frac{1}{8}[5.9375-2.1]$. Such controller is designed with the aim of achieving the prescribed specification $\frac{\delta(s)}{\delta_{d}(s)}=\frac{1.4 s+1}{s^{2}+1.5 s+1}$. 


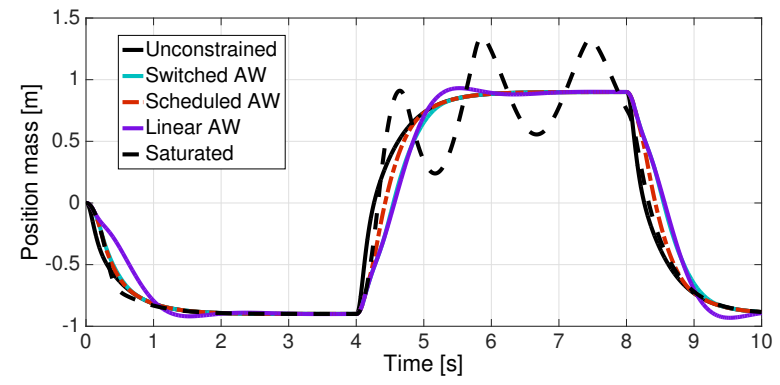

(a) Output response

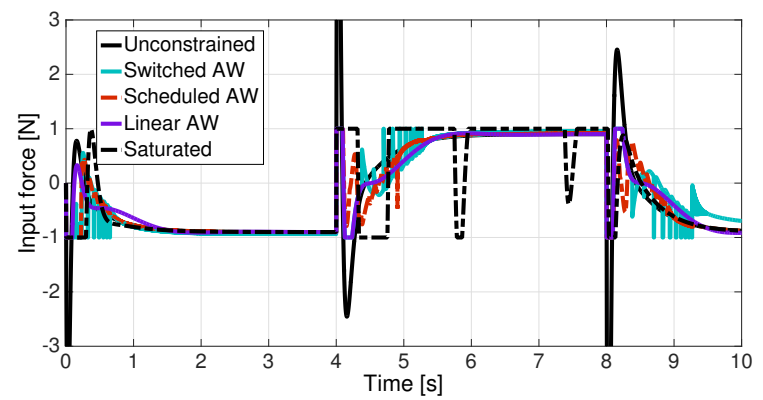

(b) Control input

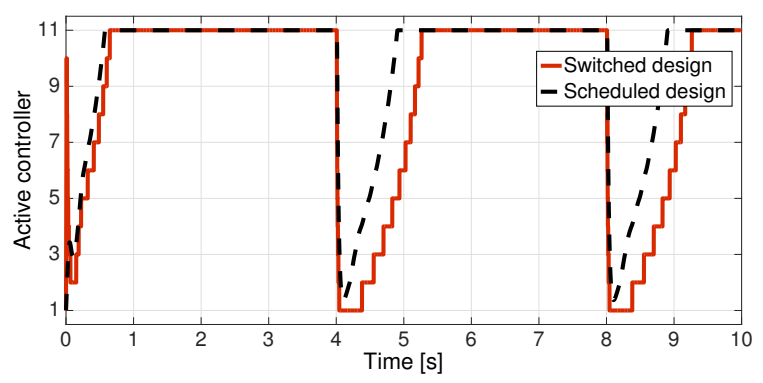

(c) Switching/Scheduling signal

Figure 5: Results for the mass-spring-damper system

Setting $\beta=0.3, \eta=0.01$ and running Algorithm 1, a family of $N=5$ nested ellipsoids is found. The corresponding exponential convergence rates are reported in Table 1. The construction of ellipsoids has been performed in Matlab using the solver SeDuMi, and the total elapsed time of computation was 8 seconds.

Table 1: Exponential rates from Algorithm 1

\begin{tabular}{|c|c|c|c|c|c|}
\hline$i$ & 1 & 2 & 3 & 4 & 5 \\
\hline$\lambda_{i}$ & 0.3447 & 3.3173 & 4.6630 & 6.7759 & 10.1065 \\
\hline
\end{tabular}

The results obtained with switched, scheduled and linear anti-windup compensators are reported in Figure 6. For the scheduled solution, the value $\ell=40$ has been used. It can be seen from the upper plot that both the switched and the scheduled scheme are characterized by improved performances with respect to the standard $\mathcal{L}_{2}$ anti-windup, while the response of the saturated plant without compensation is divergent due to exponential instability. Middle and lower plots illustrate the behavior of control inputs and switching/scheduling signals, respectively.

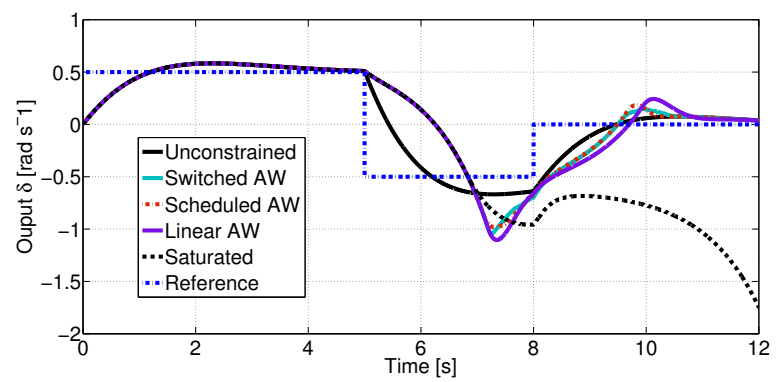

(a) Output response

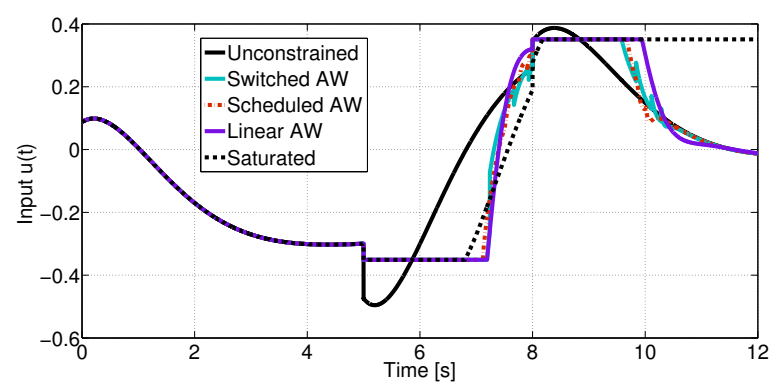

(b) Control input

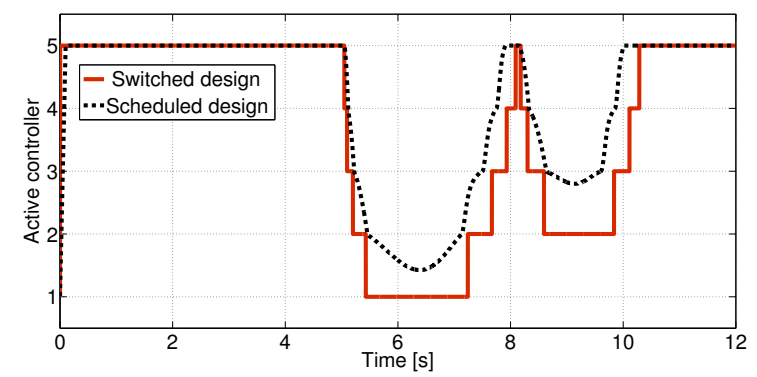

(c) Switching/Scheduling signal

Figure 6: Results for the TAFA system

\section{Conclusions}

We addressed model recovery anti-windup design with extreme performance induced by switching or scheduling antiwindup gains. The variations of the anti-windup gains was induced by a synthesis of a finite number of nested ellipsoidal sets, each of them associated to a pair of gains, and characterizing a suitable trade-off between exponential convergence rate and size of the ellipsoid. The switched solution is based on hybrid formalism involving a logic variable indicating what set of gain is active at each time, while the scheduled solution is based on a Lipschitz continuous scheduling among the finite set of available gains. Both approaches have been shown to lead to desirable closed-loop properties when combined with the antiwindup architecture, and the comparative effectiveness of the control solution has been shown on a few simulation studies. 


\section{Appendix A. Proof of Lemma 1}

The proof is adapted from [54, Prop. 1]. Let us proceed by contradiction and suppose that $\Delta \in O_{n_{u}}$ exists such that $I-S \Delta$ is singular, e.g.

$$
(I-S \Delta) z=0
$$

for some $z \neq 0$. Define $\bar{z}:=(I-S) \Delta z$; we claim that, since $z \neq 0$ and $(I-S)$ is invertible, one has necessarily $\bar{z} \neq 0$. Moreover, the following identity holds

$\bar{z}=\Delta z-S \Delta z=z+(\Delta-I) z-S \Delta z=(\Delta-I) z+(I-S \Delta) z=\Delta^{c} z$,

with $\Delta^{c}=\Delta-I$. Identity (A.1) yields $\bar{z}^{T} W(I-S \Delta) z=0$, or equivalently

$$
\bar{z}^{T} W z-\frac{1}{2} \bar{z}^{T} W S(I-S)^{-1} \bar{z}-\frac{1}{2} \bar{z}^{T}\left(I-S^{T}\right)^{-1} S^{T} W \bar{z}=0 .
$$

Observing that both $W$ and $\Delta^{c}$ are diagonal, and that the entries of $\Delta^{c}$ are $\delta_{k}^{c} \in[-1,0]$ for any $k$, one has

$$
\bar{z}^{T} W z=\sum_{k=1}^{n_{u}} \delta_{k}^{c} w_{k} z_{k}^{2} \leq-\sum_{k=1}^{n_{u}}\left(\delta_{k}^{c}\right)^{2} w_{k} z_{k}^{2}=-\bar{z}^{T} W \bar{z}
$$

and hence

$$
-\bar{z}^{T} W \bar{z}-\frac{1}{2} \bar{z}^{T} W S(I-S)^{-1} \bar{z}-\frac{1}{2} \bar{z}^{T}\left(I-S^{T}\right)^{-1} S^{T} W \bar{z} \geq 0 .
$$

However, as long as $\bar{z} \neq 0$, this contradicts the assumption $2 W+$ $W S(I-S)^{-1}+\left(I-S^{T}\right)^{-1} S^{T} W>0$.

\section{Appendix B. Proof of Lemma 3}

Set $H(x, q):=x^{T} Q^{-1}(q) x$ and observe that, by definition (30a), the mapping $H(x, q): \mathbb{R}^{n} \times \mathbb{R} \rightarrow \mathbb{R}$ is smooth everywhere except for integer values of $q$. In particular, the derivative $\frac{\partial H(x, q)}{\partial q}$ exists almost everywhere and verifies

$$
\begin{aligned}
\frac{\partial H(x, q)}{\partial q} & =x^{T} Q^{-1}(q) \frac{d Q(q)}{d q} Q^{-1}(q) x \\
& =x^{T} Q^{-1}(q)\left[Q_{\lfloor q\rfloor}-Q_{\lceil q\rceil}\right] Q^{-1}(q) x>0
\end{aligned}
$$

almost everywhere in $\mathcal{E}_{1} \backslash \mathcal{E}_{N} \times[1, N]$, because by construction $Q_{q_{1}}>Q_{q_{2}}$ for any $1 \leq q_{1}<q_{2} \leq N$ (see inequality (16c)). Then, by the nonsmooth implicit function theorem [5, Section 7.1], the equation $H(x, q)=1$ defines a unique bounded and globally Lipschitz function $q^{*}(x)$ in $\mathcal{E}_{1} \backslash \mathcal{E}_{N}$, whose gradient exists almost everywhere and satisfies the identity

$$
\begin{aligned}
\nabla_{x} q^{*}(x) & =-\left(\frac{\partial H\left(x, q^{*}(x)\right)}{\partial x}\right) /\left(\frac{\partial H\left(x, q^{*}(x)\right)}{\partial q}\right) \\
& =-\frac{2 x^{T} Q^{-1}\left(q^{*}(x)\right)}{x^{T} Q^{-1}\left(q^{*}(x)\right)\left[Q_{\left\lfloor q^{*}(x)\right\rfloor}-Q_{\left[q^{*}(x)\right]}\right] Q^{-1}\left(q^{*}(x)\right) x} .
\end{aligned}
$$

Finally one can observe that, for any integer $q \in\{1, \ldots, N\}$ and $x \in \partial \mathcal{E}_{q}$, the identity $q^{*}(x)=q$ must hold thanks to uniqueness of the inverse function.

\section{References}

[1] D. Angeli and E. Mosca. Command governors for constrained nonlinear systems. IEEE Transactions on Autotmatic Control, 44(4):816-820, April 1999.

[2] K.J. Åström and L. Rundqwist. Integrator windup and how to avoid it. In Proceedings of the American Control Conference, volume 2, pages 16931698, Pittsburgh (PA), USA, June 1989.

[3] C. Barbu, S. Galeani, A.R. Teel, and L. Zaccarian. Nonlinear anti-windup for manual flight control. Int. J. of Control, 78(14):1111-1129, September 2005 .

[4] A. Bemporad, A.R. Teel, and L. Zaccarian. Anti-windup synthesis via sampled-data piecewise affine optimal control. Automatica, 40(4):549_ 562, 2004.

[5] F.H. Clarke. Optimization and nonsmooth analysis, volume 5. Siam, 1990.

[6] JM Gomes Da Silva and Sophie Tarbouriech. Local stabilization of discrete-time linear systems with saturating controls: an lmi-based approach. IEEE Transactions on automatic control, 46(1):119-125, 2001.

[7] S. Donnarumma, L. Zaccarian, A. Alessandri, and S. Vignolo. Antiwindup synthesis of heading and speed regulators for ship control with actuator saturation. In European Control Conference, pages 1284-1290, Aalborg, Denmark, June 2016

[8] J.C. Doyle, R.S. Smith, and D.F. Enns. Control of plants with input saturation nonlinearities. In ACC, pages 1034-39, Minneapolis (MN), USA, June 1987.

[9] H.A. Fertik and C.W.Ross. Direct digital control algorithm with antiwindup feature. ISA Transactions, 6(4):317-328, 1967.

[10] F. Forni, S. Galeani, and L. Zaccarian. Model recovery anti-windup for continuous-time rate and magnitude saturated linear plants. Automatica, 48(8):1502-1513, 2012.

[11] S. Galeani, S. Onori, A.R. Teel, and L. Zaccarian. Nonlinear $\mathcal{L}_{2}$ antiwindup for enlarged stability regions and regional performance. In Symposium on Nonlinear Control Systems (NOLCOS), pages 539-544, Pretoria (South Africa), August 2007.

[12] S. Galeani, S. Onori, A.R. Teel, and L. Zaccarian. Regional, semiglobal, global nonlinear anti-windup via switching design. In European Control Conference, pages 5403-5410, Kos (Greece), July 2007.

[13] S. Galeani, S. Onori, and L. Zaccarian. Nonlinear scheduled control for linear systems subject to saturation with application to anti-windup control. In Conference on Decision and Control, pages 1168-1173, New Orleans (LA), USA, December 2007.

[14] S. Galeani, S. Tarbouriech, M.C. Turner, and L. Zaccarian. A tutorial on modern anti-windup design. European Journal of Control, 15(3-4):418440, 2009.

[15] S. Galeani, A.R. Teel, and L. Zaccarian. Constructive nonlinear antiwindup design for exponentially unstable linear plants. Systems and Control Letters, 56(5):357-365, 2007.

[16] E.G. Gilbert and I. Kolmanovsky. Fast reference governors for systems with state and control constraints and disturbance inputs. Int. J. of Robust and Nonlinear Control, 9(15):1117-1141, 1999.

[17] R. Goebel, R.G. Sanfelice, and A.R. Teel. Hybrid Dynamical Systems: modeling, stability, and robustness. Princeton University Press, 2012.

[18] J.M. Gomes da Silva and S. Tarbouriech. Anti-windup design with guaranteed regions of stability for discrete-time linear systems. Systems and Control Letters, 55(3):184-192, 2006.

[19] J.M. Gomes da Silva Jr and S. Tarbouriech. Anti-windup design with guaranteed regions of stability: an LMI-based approach. IEEE Trans. Aut. Cont., 50(1):106-111, 2005.

[20] G. Grimm, A.R. Teel, and L. Zaccarian. The $l_{2}$ anti-windup problem for discrete-time linear systems: definition and solutions. In American Control Conference, pages 5329-5334, Denver (CO), USA, June 2003.

[21] R. Hanus. Antiwindup and bumpless transfer: a survey. In IMACS World Congress, pages 59-65, Paris, France, 1988.

[22] D Henrion, G Garcia, and S Tarbouriech. Piecewise-linear robust control of systems with input constraints. European Journal of Control, 1(5):157$166,1999$.

[23] G. Herrmann, M.C. Turner, and I. Postlethwaite. Some new results on anti-windup-conditioning using the Weston-Postlethwaite approach. In IEEE Conference on Decision and Control, volume 5, pages 5047-5052, December 2004 
[24] T. Hu, Z. Lin, and B.M. Chen. An analysis and design method for linear systems subject to actuator saturation and disturbance. Automatica, 38(2):351-359, 2002.

[25] T. Hu, A.R. Teel, and L. Zaccarian. Anti-windup synthesis for linear control systems with input saturation: achieving regional, nonlinear performance. Automatica, 44(2):512-519, 2008.

[26] F. Jabbari and İ.E. Kosë. Rate and magnitude-bounded actuators: scheduled output feedback design. Int. J. of Robust and Nonlinear Control, 14:1169-1184, 2004.

[27] I. E. Kose and F. Jabbari. Scheduled controllers for linear systems with bounded actuators. Automatica, 39:1377-1387, 2003.

[28] M.V. Kothare, P.J. Campo, M. Morari, and N. Nett. A unified framework for the study of anti-windup designs. Automatica, 30(12):1869-1883, 1994.

[29] Z. Lin and A. Saberi. Low-and-high gain design technique for linear systems subject to input saturation- a direct method. Int. J. of Robust and Nonlinear Control, 7:1071-1101, 1997.

[30] A. Megretski. $\mathcal{L}_{2}$ BIBO output feedback stabilization with saturated control. In 13th Triennial IFAC World Congress, pages 435-440, San Francisco, USA, 1996.

[31] E.F. Mulder, M.V. Kothare, and M. Morari. Multivariable antiwindup controller synthesis using linear matrix inequalities. Automatica, 37(9):1407-1416, September 2001.

[32] S. Sajjadi-Kia and F. Jabbari. Multi-stage anti-windup compensation for open-loop stable plants. IEEE Transactions on Automatic Control, 56(9):2166-2172, 2011.

[33] S. Sajjadi-Kia and F. Jabbari. Controllers for linear systems with bounded actuators: Slab scheduling and anti-windup. Automatica, 49(3):762-769, 2013.

[34] E.D. Sontag. An algebraic approach to bounded controllability of linear systems. Int. J. of Control, 39(1):181-188, 1984.

[35] S. Tarbouriech, G. Garcia, J.M. Gomes da Silva Jr., and I. Queinnec. Stability and stabilization of linear systems with saturating actuators. Springer-Verlag London Ltd., 2011.

[36] S. Tarbouriech and M. Turner. Anti-windup design: an overview of some recent advances and open problems. IET Proc. Control Theory $\mathcal{G}$ Applications, 3(1):1-19, 2009.

[37] A. R. Teel and L. Praly. On assigning the derivative of a disturbance attenuation control lyapunov function. Mathematics of Control, Signals and Systems, 13(2):95-124, 2000.

[38] A.R. Teel. Global stabilization and restricted tracking for multiple integrators with bounded controls. Systems and Control Letters, 18:165-171, 1992

[39] A.R. Teel. Anti-windup for exponentially unstable linear systems. Int. J. of Robust and Nonlinear Control, 9:701-716, 1999.

[40] A.R. Teel and J.B. Buffington. Anti-windup for an F-16's daisy chain control allocator. In AIAA GNC Conference, pages 748-754, New Orleans (LA), USA, August 1997.

[41] A.R. Teel, O.E. Kaiser, and R.M. Murray. Uniting local and global controllers for the Caltech ducted fan. In Proc. of the American Control Conference, volume 3, pages 1539 -1543, Albuquerque (NM), USA, June 1997

[42] A.R. Teel and N. Kapoor. The $\mathcal{L}_{2}$ anti-windup problem: Its definition and solution. In European Control Conference, Brussels, Belgium, July 1997.

[43] A.R. Teel and N. Kapoor. Uniting local and global controllers. In European Control Conference, Brussels, Belgium, July 1997.

[44] A.R. Teel, L. Zaccarian, and J. Marcinkowski. An anti-windup strategy for active vibration isolation systems. Control Engineering Practice, 14(1):17-27, 2006.

[45] F. Todeschini, S. Formentin, G. Panzani, M. Corno, S. Savaresi, and L. Zaccarian. Nonlinear pressure control for BBW systems via dead zone and anti-windup compensation. IEEE Transactions on Control Systems Technology, (4):1419-1431, 2016.

[46] M.C. Turner and G. Herrmann. A non-square sector condition and its application in deferred-action anti-windup compensator design. Automatica, 50(1):268-276, 2014.

[47] M.C. Turner, J. Sofrony, and G. Herrmann. An alternative approach to anti-windup in anticipation of actuator saturation. International Journal of Robust and Nonlinear Control, 27(6):963-980, 2017. rnc.3609.

[48] G. Valmorbida, L. Zaccarian, S. Tarbouriech, I. Queinnec, and A. Papachristodoulou. Nonlinear static state feedback for saturated linear plants via a polynomial approach. IEEE Transactions on Automatic Control, (1):469-474, 2017.

[49] P. Weston and I. Postlethwaite. Linear conditioning schemes for systems containing saturating actuators. In 4th Nonlinear control systems design symposium (NOLCOS), pages 702-707, Enschede, The Netherlands, July 1998.

[50] GF Wredenhagen and PR Belanger. Piecewise-linear lq control for systems with input constraints. Automatica, 30(3):403-416, 1994

[51] X. Wu and Z. Lin. On immediate, delayed and anticipatory activation of anti-windup mechanism: static anti-windup case. IEEE Transactions on Automatic Control, 57(3):771-777, 2012.

[52] X. Wu and Z. Lin. Dynamic anti-windup design in anticipation of actuator saturation. International Journal of Robust and Nonlinear Control, 24(2):295-312, 2014.

[53] L. Zaccarian, D. Nešić, and A.R. Teel. $\mathcal{L}_{2}$ anti-windup for linear deadtime systems. Systems and Control Letters, 54(12):1205-1217, 2005.

[54] L. Zaccarian and A.R. Teel. A common framework for anti-windup, bumpless transfer and reliable designs. Automatica, 38(10):1735-1744, 2002.

[55] L. Zaccarian and A.R. Teel. Nonlinear scheduled anti-windup design for linear systems. IEEE Transactions on Automatic Control, 49(11):20552061, 2004.

[56] L. Zaccarian and A.R. Teel. The $\mathcal{L}_{2}\left(l_{2}\right)$ bumpless transfer problem: its definition and solution. Automatica, 41(7):1273-1280, 2005.

[57] L. Zaccarian and A.R. Teel. Modern anti-windup synthesis: control augmentation for actuator saturation. Princeton University Press, Princeton (NJ), 2011.

[58] L. Zaccarian, E. Weyer, A.R. Teel, Y. Li, and M. Cantoni. Anti-windup for marginally stable plants and its application to open water channel control systems. Control Engineering Practice, 15(2):261-272, 2007. 